\begin{tabular}{|c|l|}
\hline Title & A Database Schema for the A nalysis of Global Dynamics of Multiparameter Systems \\
\hline Author(s) & A rai, Zin; Kalies, William; Kokubu, Hiroshi; Mischaikow, Konstantin; Oka, Hiroe; Pilarczyk, Pawet \\
\hline Citation & $\begin{array}{l}\text { SIAM Journal on A pplied Dynamical Sy stems, 8(3), 757-789 } \\
\text { https://doi.org/10.1137/080734935 }\end{array}$ \\
\hline Issue Date & 2009-07-02 \\
\hline Doc URL & http://hdl.handle.net/2115/39480 \\
\hline Rights & ○ 2009 Society for Industrial and A pplied Mathematics \\
\hline Type & article \\
\hline File Information & JADS8_3_p757-789.pdf \\
\hline
\end{tabular}

Instructions for use 


\title{
A Database Schema for the Analysis of Global Dynamics of Multiparameter Systems*
}

\author{
Zin Arai ${ }^{\dagger}$, William Kalies ${ }^{\ddagger}$, Hiroshi Kokubu ${ }^{\S}$ Konstantin Mischaikow , Hiroe Okall, and \\ Paweł Pilarczyk**
}

Abstract. A generally applicable, automatic method for the efficient computation of a database of global dynamics of a multiparameter dynamical system is introduced. An outer approximation of the dynamics for each subset of the parameter range is computed using rigorous numerical methods and is represented by means of a directed graph. The dynamics is then decomposed into the recurrent and gradient-like parts by fast combinatorial algorithms and is classified via Morse decompositions. These Morse decompositions are compared at adjacent parameter sets via continuation to detect possible changes in the dynamics. The Conley index is used to study the structure of isolated invariant sets associated with the computed Morse decompositions and to detect the existence of certain types of dynamics. The power of the developed method is illustrated with an application to the two-dimensional density-dependent Leslie population model. An interactive visualization of the results of computations discussed in the paper can be accessed at the Web site http://chomp.rutgers.edu/database/, and the source code of the software used to obtain these results has also been made freely available.

Key words. database, dynamical system, Conley index, Morse decomposition, Leslie population models, combinatorial dynamics, multiparameter system

AMS subject classifications. Primary, 37B35; Secondary, 37B30, 37M99, 37N25, 92-08

DOI. $10.1137 / 080734935$

\footnotetext{
${ }^{*}$ Received by the editors September 9, 2008; accepted for publication (in revised form) by T. Kaper April 2, 2009; published electronically July 2, 2009.

http://www.siam.org/journals/siads/8-3/73493.html

†PRESTO, Japan Science and Technology Agency/Hokkaido University, Creative Research Institute "Sousei," Sapporo 001-0021, Japan (arai@cris.hokudai.ac.jp). The work of this author was partially supported by Grant-in-Aid for Scientific Research 17740054, Ministry of Education, Science, Technology, Culture and Sports, Japan.

‡Department of Mathematical Sciences, Florida Atlantic University, 777 Glades Rd., Boca Raton, FL 33431 (wkalies@fau.edu). The work of this author was partially supported by NSF grant DMS-0511208 and DOE grant DE-FG02-05ER25713.

${ }^{\S}$ Department of Mathematics, Kyoto University, Kyoto 606-8502, Japan (kokubu@math.kyoto-u.ac.jp). The work of this author was partially supported by Grant-in-Aid for Scientific Research 17340045, Ministry of Education, Science, Technology, Culture and Sports, Japan.

"Department of Mathematics and BioMaPS, Hill Center-Busch Campus, Rutgers, The State University of New Jersey, 110 Frelinghuysen Rd., Piscataway, NJ 08854-8019 (mischaik@math.rutgers.edu). The work of this author was partially supported by NSF grant DMS-0511115, DARPA, and DOE grant DE-FG02-05ER25711.

"Department of Applied Mathematics and Informatics, Faculty of Science and Technology, Ryukoku University, Seta, Otsu 520-2194, Japan (oka@rins.ryukoku.ac.jp). The work of this author was partially supported by Grant-inAid for Scientific Research 17540206, Ministry of Education, Science, Technology, Culture and Sports, Japan.

${ }^{* *}$ Centre of Mathematics, University of Minho, Campus de Gualtar, 4710-057 Braga, Portugal, and Department of Mathematics, Kyoto University, Kyoto 606-8502, Japan (pawelpil@math.kyoto-u.ac.jp). The work of this author was partially supported by the JSPS Postdoctoral Fellowship P06039 and by Grant-in-Aid for Scientific Research 1806039, Ministry of Education, Science, Technology, Culture and Sports, Japan.
}

757

Copyright (C) by SIAM. Unauthorized reproduction of this article is prohibited. 
1. Introduction. The global dynamics of a nonlinear system can exhibit structures at all spatial scales, for example, the fractal structures associated to chaotic dynamics. The same phenomenon can occur with respect to the parameters; that is, global dynamical structures can change on Cantor sets in parameter space. From the point of view of scientific computation, only a finite amount of information can be computed, and therefore, any computational characterization of global dynamics of a multiparameter system can be expected to represent a dramatic reduction of information. Nevertheless, the computation of global dynamical information is an important problem for applications, which leads to the questions of how to characterize global dynamical structures and how to identify changes in these structures in practice. The fact that this is a nontrivial task has been made clear by the work of the dynamical systems community over the last century.

Identifying and classifying the qualitative properties of models over wide ranges of parameter values is of fundamental importance in many disciplines, and in particular it is of primary interest to many computational biologists [10]. The fact that most topics of interest in systems biology are dynamic in nature suggests the need for a comprehensive yet efficient method for cataloguing the global dynamics of nonlinear systems. In other words, a method is desired which computationally constructs a database of global dynamical behavior of a specific system over a range of parameters.

The starting point for our computational methods is Conley's topological approach to dynamics [2], which we review in the next section. The prior work in $[1,5,9,12,24]$ has shown that Conley theory is an appropriate theoretical base for designing algorithms in computational dynamics. The purpose of this paper is to demonstrate that these ideas can be used to design an efficient computational framework for constructing databases of global dynamics of specific systems over multiple parameters. While the methods we propose are general, we illustrate them using a particular population model which we describe in section 1.2.

1.1. Preliminary ideas. We restrict our attention to the setting of a multiparameter family of dynamical systems given by a continuous function

$$
f: X \times \Lambda \ni(x, \lambda) \mapsto f(x, \lambda)=f_{\lambda}(x) \in X,
$$

where the phase space $X$ is a locally compact metric space and the parameter space $\Lambda$ is a compact, locally contractible, connected metric space. Note that we do not assume that $f_{\lambda}$ is a homeomorphism.

The fundamental structures in any dynamical system are the invariant sets. A set $Z \subset X$ is invariant at $\lambda \in \Lambda$ if $f_{\lambda}(Z)=Z$. Since we cannot perform computations at each parameter value independently, we are interested in considering sets which are invariant with respect to a subset of the parameter space. We use the notation $F: X \times \Lambda \rightarrow X \times \Lambda$ for the trivial extension of the system to include the parameters as explicit variables defined by

$$
F(x, \lambda)=\left(f_{\lambda}(x), \lambda\right)=(f(x, \lambda), \lambda) .
$$

For $\Lambda_{0} \subset \Lambda$ we denote the restriction of $F$ to $X \times \Lambda_{0}$ by $F_{\Lambda_{0}}: X \times \Lambda_{0} \rightarrow X \times \Lambda_{0}$. Observe that $F=F_{\Lambda}$ and that $f_{\lambda}$ is readily identified with $F_{\{\lambda\}}$. Moreover, given a set $S \subset X \times \Lambda$, we denote its restriction to $\Lambda_{0}$ by $S_{\Lambda_{0}}:=S \cap\left(X \times \Lambda_{0}\right)$. In particular, we often identify $S_{\lambda} \subset X$ with

Copyright (c) by SIAM. Unauthorized reproduction of this article is prohibited. 
$S_{\{\lambda\}}=S_{\lambda} \times\{\lambda\}$. In this language, a set $S \subset X \times \Lambda$ is invariant over $\Lambda_{0}$ if $F_{\Lambda_{0}}\left(S_{\Lambda_{0}}\right)=S_{\Lambda_{0}}$, which is an equivalent way of stating that the set $S_{\lambda}$ is invariant at all $\lambda \in \Lambda_{0}$.

We wish to identify and characterize a finite collection of invariant sets which determine the global, qualitative behavior of the dynamics. In addition, we need a method for comparing two invariant sets $S_{\lambda_{0}}$ and $S_{\lambda_{1}}$ at distinct parameters. Deciding what sets to compute and how to compare them are the fundamental issues we attempt to address.

We emphasize that while the amount of information that can be computed is finite, this does not mean that we cannot detect or characterize dynamical structures that occur on arbitrarily small scales. For example, topological techniques can be used to identify chaotic dynamics via a semiconjugacy onto a subshift of finite type; see [12]. Moreover, even though we cannot compute the dynamics at each parameter separately, this does not mean that we cannot obtain results which apply to every parameter value. Indeed, the methods we employ do compute mathematically rigorous global structures for invariant sets at all parameter values.

1.2. Model example. To provide perspective on the practicality and utility of our approach, we consider the two-dimensional version of an overcompensatory Leslie population model $g: \mathbb{R}^{2} \times \mathbb{R}^{4} \rightarrow \mathbb{R}^{2}$ given by

$$
\left[\begin{array}{l}
x_{1} \\
x_{2}
\end{array}\right] \mapsto\left[\begin{array}{c}
\left(\theta_{1} x_{1}+\theta_{2} x_{2}\right) e^{-\phi\left(x_{1}+x_{2}\right)} \\
p x_{1}
\end{array}\right]
$$

where the fertility rates decay exponentially with population size. This model and its biological relevance is discussed in considerable detail in work of Ugarcovici and Weiss [25]. As indicated there, in view of $g(x, \theta, p, \phi)=\phi^{-1} g(\phi x, \theta, p, 1)$, the constant $\phi$ may be scaled arbitrarily, and thus it suffices to study $f: \mathbb{R}^{2} \times \mathbb{R}^{3} \rightarrow \mathbb{R}^{2}$ given by

$$
(x, \lambda)=\left(\left[\begin{array}{l}
x_{1} \\
x_{2}
\end{array}\right],\left[\begin{array}{c}
\theta_{1} \\
\theta_{2} \\
p
\end{array}\right]\right) \mapsto f(x, \lambda)=\left[\begin{array}{c}
\left(\theta_{1} x_{1}+\theta_{2} x_{2}\right) e^{-0.1\left(x_{1}+x_{2}\right)} \\
p x_{1}
\end{array}\right] .
$$

Furthermore, the detailed numerical studies of [25] indicate that this system exhibits a wide variety of different dynamical behavior, which suggests that it provides a meaningful test for the usefulness of the techniques introduced in this paper.

1.3. Outline of the paper. In the remainder of the paper we develop our method and apply it to the Leslie model. In section 2 we introduce the dynamical structures which we utilize and review Conley theory. In section 3 we introduce a method for building a combinatorial representation of the dynamics and a means of comparing the computed structures at different parameters. In section 4 we address the question of efficient computational algorithms based on rectangular grids in $X$ and $\Lambda$. In section 5 we describe a database computed with our methods for the model example introduced in section 1.2 with $p=0.7$ and $\theta_{1}, \theta_{2}$ varying in selected ranges, and we show how this database can be queried for various dynamical properties. Finally, in section 6 we briefly mention a result containing similar computations, where all three parameters are varied.

Copyright (c) by SIAM. Unauthorized reproduction of this article is prohibited. 
2. Review of Conley theory. Recall that a compact set $N \subset X \times \Lambda_{0}$ is an isolating neighborhood for $F_{\Lambda_{0}}$ if

$$
\operatorname{Inv}\left(N, F_{\Lambda_{0}}\right) \subset \operatorname{int}_{X \times \Lambda_{0}}(N),
$$

where $\operatorname{Inv}\left(N, F_{\Lambda_{0}}\right)$ denotes the maximal invariant set in $N$ under $F_{\Lambda_{0}}$, and $\operatorname{int}_{X \times \Lambda_{0}}(N)$ denotes the interior of $N$ with respect to the subspace topology on $X \times \Lambda_{0}$. Isolating neighborhoods are computable and hence provide a means of identifying invariant sets. In particular, an invariant set $S_{\Lambda_{0}} \subset X \times \Lambda_{0}$ is an isolated invariant set if $S_{\Lambda_{0}}=\operatorname{Inv}\left(N, F_{\Lambda_{0}}\right)$ for some isolating neighborhood $N$.

As indicated in $[2,15]$, the space of isolated invariant sets is a sheaf. More precisely, if $N$ is an isolating neighborhood for $F=F_{\Lambda}$ and $S=\operatorname{Inv}(N, F)$, then for any $\Lambda_{0} \subset \Lambda, N_{\Lambda_{0}}$ is an isolating neighborhood for $F_{\Lambda_{0}}$ and $S_{\Lambda_{0}}=\operatorname{Inv}\left(N_{\Lambda_{0}}, F_{\Lambda_{0}}\right)$. In particular, for any $\lambda \in \Lambda$, $S_{\{\lambda\}} \subset X \times\{\lambda\}$ is an isolated invariant set for $F_{\{\lambda\}}: X \times\{\lambda\} \rightarrow X \times\{\lambda\}$ and $S_{\lambda} \subset X$ is an isolated invariant set for $f_{\lambda}: X \rightarrow X$.

To simplify the presentation and analysis, throughout this paper we make use of the following assumption and establish the following notation.

A1: There exists a compact set $B \subset X \times \Lambda$ which is an isolating neighborhood for $F$. Its maximal invariant set is denoted by $S:=\operatorname{Inv}(B, F)$.

Using the sheaf property of isolated invariant sets, $S_{\Lambda_{0}}$ denotes the restriction of $S$ to $X \times \Lambda_{0}$, which is an isolated invariant set for $F_{\Lambda_{0}}$ if $\Lambda_{0}$ is compact. As indicated above, we are interested in understanding the structure of $S_{\lambda}$ for all $\lambda \in \Lambda$, and we make use of two essential ideas due to Conley [2] — Morse decompositions and the Conley index.

2.1. Morse decompositions. Recall that a Morse decomposition of $S_{\Lambda_{0}}$ is a finite collection

$$
\mathbf{M}\left(S_{\Lambda_{0}}\right)=\left\{M_{\Lambda_{0}}(p) \subset S_{\Lambda_{0}} \mid p \in \mathcal{P}_{\Lambda_{0}}\right\}
$$

of disjoint isolated invariant sets of $F_{\Lambda_{0}}$, called Morse sets, which are indexed by the set $\mathcal{P}_{\Lambda_{0}}$ on which there exists a strict partial order $>_{\Lambda_{0}}$, called an admissible order, such that for every $(x, \lambda) \in S_{\Lambda_{0}} \backslash \bigcup_{p \in \mathcal{P}} M_{\Lambda_{0}}(p)$ and any complete orbit $\gamma$ of $F_{\Lambda_{0}}$ through $(x, \lambda)$ in $S_{\Lambda_{0}}$ there exist indices $p>_{\Lambda_{0}} q$ such that under $F_{\Lambda_{0}}$

$$
\omega(\gamma) \subset M_{\Lambda_{0}}(q) \text { and } \quad \alpha(\gamma) \subset M_{\Lambda_{0}}(p)
$$

Morse decompositions provide a coarse but global description of the dynamics on $S_{\Lambda_{0}}$.

Remark 2.1. With regard to the construction of a database for global dynamics, we make several important observations concerning Morse decompositions; see [2, 13].

1. Morse decompositions of $S_{\Lambda_{0}}$ are not unique. They can often be refined or coarsened, and many systems have no finest Morse decomposition.

2. The empty set can be a Morse set.

3. Every structure within $S_{\Lambda_{0}}$ that is associated with recurrent dynamics, e.g., a fixed point, a periodic orbit, or chaotic dynamics, must lie in some Morse set. Away from the Morse sets the dynamics is gradient-like, and the direction of trajectories is captured by an admissible partial order. 
4. Given a Morse decomposition, there is a unique minimal admissible partial order called the flow-defined order. Any extension of the flow-defined order which maintains a strict partial order produces an admissible order.

5. Consider a Morse decomposition $\mathbf{M}\left(S_{\Lambda_{0}}\right)=\left\{M_{\Lambda_{0}}(p) \subset S_{\Lambda_{0}} \mid p \in \mathcal{P}_{\Lambda_{0}}\right\}$ of $S_{\Lambda_{0}}$ with admissible order $>_{\Lambda_{0}}$. If $\Lambda_{1} \subset \Lambda_{0}$, then the collection of sets

$$
\left\{M_{\Lambda_{1}}(p) \subset S_{\Lambda_{1}} \mid p \in \mathcal{P}_{\Lambda_{0}}\right\},
$$

where $M_{\Lambda_{1}}(p):=M_{\Lambda_{0}}(p) \cap\left(X \times \Lambda_{1}\right)$, is a Morse decomposition of $S_{\Lambda_{1}}$ under $F_{\Lambda_{1}}$ with the same admissible order.

Observe that since $\mathcal{P}_{\Lambda_{0}}$ is a strict partially ordered set, a Morse decomposition can be represented as an acyclic directed graph $\mathrm{MG}\left(\Lambda_{0}\right)$ called the Morse graph over $\Lambda_{0}$. The elements of the index set $\mathcal{P}_{\Lambda_{0}}$, which naturally correspond to the Morse sets, are the vertices of the Morse graph over $\Lambda_{0}$, and the edges of the Morse graph over $\Lambda_{0}$ are the minimal order relations which through transitivity generate $>_{\Lambda_{0}}$. In other words, for $p, q \in \mathcal{P}_{\Lambda_{0}}$ there is a directed edge $p \rightarrow q$ in MG( $\left.\Lambda_{0}\right)$ if $p>\Lambda_{0} q$ and there does not exist $r \in \mathcal{P}_{\Lambda_{0}}$ such that $p>_{\Lambda_{0}} r>_{\Lambda_{0}} q$.

Computationally, we obtain Morse decompositions indirectly, and often it can be established by further computation that a Morse set is empty. In that case, such a set can be removed from the Morse decomposition, as described by the following proposition, whose proof follows directly from the definition of a Morse decomposition.

Proposition 2.2. Consider a Morse decomposition $\mathbf{M}\left(S_{\Lambda_{0}}\right)=\left\{M_{\Lambda_{0}}(p) \subset S_{\Lambda_{0}} \mid p \in \mathcal{P}_{\Lambda_{0}}\right\}$ of $S_{\Lambda_{0}}$ with admissible order $>$. Assume $M_{\Lambda_{0}}\left(p_{0}\right)=\emptyset$. Then

$$
\mathbf{M}^{\prime}\left(S_{\Lambda_{0}}\right):=\left\{M_{\Lambda_{0}}(p) \subset S_{\Lambda_{0}} \mid p \in \mathcal{P}_{\Lambda_{0}} \backslash\left\{p_{0}\right\}\right\}
$$

is a Morse decomposition of $S_{\Lambda_{0}}$ and an admissible order is given by $>^{\prime}$ where for all $p, q \in$ $\mathcal{P}_{\Lambda_{0}} \backslash\left\{p_{0}\right\}$ we have $p>^{\prime} q$ if and only if $p>q$.

From Proposition 2.2, if $\mathrm{MG}\left(\Lambda_{0}\right)$ is the Morse graph over $\Lambda_{0}$ corresponding to $\mathbf{M}\left(S_{\Lambda_{0}}\right)$, then $\mathrm{MG}^{\prime}\left(\Lambda_{0}\right)$, the Morse graph over $\Lambda_{0}$ corresponding to $\mathbf{M}^{\prime}\left(S_{\Lambda_{0}}\right)$, has vertices $\mathcal{P}_{\Lambda_{0}} \backslash\left\{p_{0}\right\}$ and has an edge $p \rightarrow q$ if either $p \rightarrow q$ is an edge in $\operatorname{MG}\left(\Lambda_{0}\right)$ or $p \rightarrow p_{0} \rightarrow q$ are edges in $\operatorname{MG}\left(\Lambda_{0}\right)$. The simplified Morse graph $\mathrm{MG}^{\prime}\left(\Lambda_{0}\right)$ is called a trivial reduction of $\mathrm{MG}\left(\Lambda_{0}\right)$. Whenever possible, we work with a trivial reduction of a Morse graph. The possibility that a Morse set is trivial can be detected via the Conley index, which is described in the next section.

2.2. The Conley index. As explained in section 3, computational methods exist to find Morse decompositions; see [1]. The Conley index, which is an algebraic topological invariant of isolated invariant sets, is used to understand the structure of the dynamics within a Morse set.

To explain the index we begin by considering an arbitrary continuous map $g: Z \rightarrow Z$ on a locally compact metric space and a pair of compact sets $N=\left(N_{1}, N_{0}\right)$ such that $N_{0} \subset N_{1} \subset Z$. Consider the pointed quotient space $\left(N_{1} / N_{0},\left[N_{0}\right]\right)$ obtained by collapsing $N_{0}$ to a single point $\left[N_{0}\right]$. Define $g_{N}:\left(N_{1} / N_{0},\left[N_{0}\right]\right) \rightarrow\left(N_{1} / N_{0},\left[N_{0}\right]\right)$ by

$$
g_{N}(x)= \begin{cases}g(x) & \text { if } x, g(x) \in N_{1} \backslash N_{0} \\ {\left[N_{0}\right]} & \text { otherwise. }\end{cases}
$$

Copyright $\odot$ by SIAM. Unauthorized reproduction of this article is prohibited. 
The pair $N=\left(N_{1}, N_{0}\right)$ is an index pair if the map $g_{N}$ is continuous and $\operatorname{cl}\left(N_{1} \backslash N_{0}\right)$ is an isolating neighborhood [21].

The following two facts about index pairs are most relevant and can be found in $[2,13]$.

1. For any isolated invariant set $K$, there exists at least one index pair $N=\left(N_{1}, N_{0}\right)$ such that $K=\operatorname{Inv}\left(\operatorname{cl}\left(N_{1} \backslash N_{0}\right), g\right)$.

2. For any isolated invariant set $K$, there can exist many index pairs which isolate $K$.

The first fact implies that for any Morse set $M_{\Lambda_{0}}(p)$ for the system $F_{\Lambda_{0}}$ there exists an index pair $N=\left(N_{1}, N_{0}\right)$ such that the induced map $F_{\Lambda_{0}, N}:\left(N_{1} / N_{0},\left[N_{0}\right]\right) \rightarrow\left(N_{1} / N_{0},\left[N_{0}\right]\right)$ is a continuous function and $M_{\Lambda_{0}}(p)=\operatorname{Inv}\left(\operatorname{cl}\left(N_{1} \backslash N_{0}\right), F_{\Lambda_{0}}\right)$. Passing to homology leads to a family of group endomorphisms

$$
F_{\Lambda_{0}, N *}: H_{*}\left(N_{1} / N_{0},\left[N_{0}\right]\right) \rightarrow H_{*}\left(N_{1} / N_{0},\left[N_{0}\right]\right) .
$$

The second fact allows for different choices of index pairs which can lead to different group endomorphisms. Thus, to define the Conley index of an isolated invariant set, such as a Morse set $M_{\Lambda_{0}}(p)$, one must consider equivalence classes of these group endomorphisms [13]. In constructing our database, we do not utilize the full Conley index; instead, we store a weaker invariant, namely, the nonzero eigenvalues of $F_{\Lambda_{0}, N *}$ restricted to the torsion-free part of $H_{*}\left(N_{1} / N_{0},\left[N_{0}\right]\right)$. This weaker invariant is chosen because these eigenvalues are readily computed and compared.

Remark 2.3. With regard to the construction and interpretation of the database, there are several important observations about the index to be made; see [13].

1. If the Conley index is trivial, then the map $F_{\Lambda_{0}, N *}$ is nilpotent. This implies that the eigenvalues of $F_{\Lambda_{0}, N *}$ restricted to the torsion-free part of $H_{*}\left(N_{1} / N_{0},\left[N_{0}\right]\right)$ are all zero.

2. The empty set is an isolated invariant set with trivial Conley index. Thus, if there are nonzero eigenvalues of $F_{\Lambda_{0}, N *}$ restricted to the torsion-free part of $H_{*}\left(N_{1} / N_{0},\left[N_{0}\right]\right)$, then $M_{\Lambda_{0}}(p) \neq \emptyset$.

3. There exist nontrivial isolated invariant sets with trivial Conley index. For example, consider the logistic map $g_{\lambda}(x)=\lambda x(1-x), \lambda \in[1,4]$, and an index pair $N=$ $\left(N_{1}, N_{0}\right)=([-1,2],\{-1,2\})$. The index map $F_{N *}$ is nilpotent, but clearly $S_{\lambda}=$ $\operatorname{Inv}\left(N_{1} \backslash N_{0}, g_{\lambda}\right) \neq \emptyset$.

4. The Conley index can be used to reconstruct some of the structure of the dynamics of the associated isolated invariant set. In particular, under appropriate conditions the Conley index can be used to conclude the existence of fixed points, periodic orbits, and chaotic dynamics.

5. Suppose $\Lambda_{1} \subset \Lambda_{0}$ are both contractible. If $M_{\Lambda_{0}}(p)$ is a Morse set, or indeed any isolated invariant set, then the nonzero eigenvalues of the torsion-free part of any index map $F_{\Lambda_{0}, N *}$ are identical to the nonzero eigenvalues of the torsion-free part of any index map $F_{\Lambda_{1}, N *}$ for the set $M_{\Lambda_{1}}(p)=M_{\Lambda_{0}}(p) \cap\left(X \times \Lambda_{1}\right)$.

2.3. Conley-Morse graphs. We are now in a position to describe the fundamental element of our database. Let $\Lambda_{0} \subset \Lambda$, and let $\mathbf{M}\left(S_{\Lambda_{0}}\right)=\left\{M_{\Lambda_{0}}(p) \subset S_{\Lambda_{0}} \mid p \in \mathcal{P}_{\Lambda_{0}}\right\}$ be a Morse decomposition of $S_{\Lambda_{0}}$ with admissible order $>\Lambda_{0}$. The Conley-Morse graph over $\Lambda_{0}$ of $\mathbf{M}\left(S_{\Lambda_{0}}\right)$ is denoted by $\operatorname{CMG}\left(\Lambda_{0}\right)$ and consists of $\operatorname{MG}\left(\Lambda_{0}\right)$, the Morse graph over $\Lambda_{0}$ with the additional

Copyright $\odot$ by SIAM. Unauthorized reproduction of this article is prohibited. 
information of the nonzero eigenvalues of the torsion-free part of the index map of each Morse set assigned to the associated node. We present this information by labeling each node in the following format.

$$
\mathrm{p} k: n \rightarrow\{*\}
$$

denotes the fact that the $k$ th Morse set has nonzero eigenvalues $\{*\}$ on the $n$th level of homology. If the $k$ th Morse set has no nonzero eigenvalues, then we write

$$
\mathrm{p} k: 0 \text {. }
$$

An example of how this information can be used to describe the structure of the dynamics is given in Proposition 5.8.

As described in sections 3 and 4, we compute the Conley-Morse graphs over distinct fixed subregions of parameter space. This raises the question of how to relate the resulting Conley-Morse graphs. One answer is provided in the following definition.

Definition 2.4. Assume A1. Let $\Lambda_{0}, \Lambda_{1} \subset \Lambda$ be such that $\Lambda_{0,1}:=\Lambda_{0} \cap \Lambda_{1}$ is a nonempty, contractible set. Let

$$
\mathbf{M}\left(S_{\Lambda_{i}}\right)=\left\{M_{\Lambda_{i}}(p) \subset S_{\Lambda_{i}} \mid p \in \mathcal{P}_{\Lambda_{i}}\right\}
$$

for $i=0,1$ be Morse decompositions with admissible orders $>_{i}$. The associated Morse graphs $\operatorname{MG}\left(\Lambda_{0}\right)$ over $\Lambda_{0}$ and $\mathrm{MG}\left(\Lambda_{1}\right)$ over $\Lambda_{1}$ are equivalent if there exists an order preserving bijection $\iota:\left(\mathcal{P}_{\Lambda_{0}},>_{0}\right) \rightarrow\left(\mathcal{P}_{\Lambda_{1}},>_{1}\right)$ such that

$$
M_{\Lambda_{0}}(p) \cap\left(X \times \Lambda_{0,1}\right)=M_{\Lambda_{1}}(\iota(p)) \cap\left(X \times \Lambda_{0,1}\right) .
$$

The continuation property of the Conley index [2] implies that if $\operatorname{MG}\left(\Lambda_{0}\right)$ and $\operatorname{MG}\left(\Lambda_{1}\right)$ are equivalent via the order preserving bijection $\iota:\left(\mathcal{P}_{\Lambda_{0}},>_{0}\right) \rightarrow\left(\mathcal{P}_{\Lambda_{1}},>_{1}\right)$, then the ConleyMorse graphs $\operatorname{CMG}\left(\Lambda_{0}\right)$ and $\operatorname{CMG}\left(\Lambda_{1}\right)$ are also equivalent; that is, the nonzero eigenvalues associated to corresponding Morse sets are identical.

3. Combinatorial representation of dynamics. Conley-Morse graphs are the key elements of information stored in our database. In this section we describe a general procedure for computing these graphs. We begin by describing a means of combinatorializing the dynamics.

Recall [17] that a grid on a metric space $Z$ is a collection $\mathcal{Z}$ of nonempty, compact subsets of $Z$ with the following properties:

(a) $Z=\bigcup_{G \in \mathcal{Z}} G$,

(b) $G=\operatorname{cl}(\operatorname{int}(G))$ for all $G \in \mathcal{Z}$,

(c) $G \cap \operatorname{int}(H)=\emptyset$ for all $G \neq H \in \mathcal{Z}$, and

(d) if $K \subset Z$ is compact, then $\{G \in \mathcal{Z} \mid G \cap K \neq \emptyset\}$ is a finite set.

The grid $\mathcal{Z}$ has the simple intersection property if $G, H \in \mathcal{Z}$ and $G \cap H \neq \emptyset$ implies that $G \cap H$ is contractible. The diameter of a grid is defined by $\operatorname{diam}(\mathcal{Z}):=\sup _{G \in \mathcal{Z}} \operatorname{diam}(G)$, and the realization map $|\cdot|$ is a function from subsets of $\mathcal{Z}$ to subsets of $Z$ defined by $|\mathcal{A}|:=\bigcup_{A \in \mathcal{A}} A$. Given $Y \subset Z$, define

$$
\mathcal{Z}(Y):=\{G \in \mathcal{Z} \mid \operatorname{int}(G) \cap Y \neq \emptyset\}
$$

Copyright $@$ by SIAM. Unauthorized reproduction of this article is prohibited. 
For the remainder of the paper, $\mathcal{X}$ and $\mathcal{Q}$ denote grids with the simple intersection property on $X$ and $\Lambda$, respectively. Observe that $\mathcal{X} \times \mathcal{Q}$ is a grid for $X \times \Lambda$. As shown in [9], given $\delta>0$, we can choose grids such that $\operatorname{diam}(\mathcal{X})<\delta$ and $\operatorname{diam}(\mathcal{Q})<\delta$.

A combinatorial multivalued map $\mathcal{F}: \mathcal{Z} \rightrightarrows \mathcal{Z}$ assigns to each element $G \in \mathcal{Z}$ a finite (possibly empty) subset $\mathcal{F}(G)$ of $\mathcal{Z}$. Important for efficient computation is the observation that a combinatorial multivalued map $\mathcal{F}: \mathcal{Z} \rightrightarrows \mathcal{Z}$ is equivalent to a directed graph with vertices $\mathcal{Z}$ and directed edges $(G, H)$ whenever $H \in \mathcal{F}(G)$. A directed graph is closed if each vertex is both the head of at least one edge and the tail of at least one edge.

We use combinatorial multivalued maps on the grid $\mathcal{X}$ as a means to discretize and combinatorially approximate the dynamics of $f$. For a more detailed description, see [9]. Fix $\lambda \in \Lambda$ and a compact set $B_{\lambda} \subset X$. We relate $\left.f_{\lambda}\right|_{B_{\lambda}}: B_{\lambda} \rightarrow X$ to the combinatorial multivalued map $\mathcal{F}_{\lambda}: \mathcal{X}\left(B_{\lambda}\right) \rightrightarrows \mathcal{X}$ by requiring that $\mathcal{F}_{\lambda}$ outer approximates $f_{\lambda}$ in the following sense [24]. A multivalued map $\mathcal{F}_{\lambda}: \mathcal{X}\left(B_{\lambda}\right) \rightrightarrows \mathcal{X}$ is called an outer approximation of $f_{\lambda}$ restricted to $\left|\mathcal{X}\left(B_{\lambda}\right)\right|$ if $f_{\lambda}(G) \subset \operatorname{int}\left(\left|\mathcal{F}_{\lambda}(G)\right|\right)$ for all $G \in \mathcal{X}\left(B_{\lambda}\right)$.

The property of being an outer approximation is the key to approximating the dynamics of $f$ combinatorially. Since we can perform only a finite number of computations, we cannot compute $\mathcal{F}_{\lambda}$ individually for each $\lambda \in \Lambda$. Recall that we have made the assumption A1 so that the compact set $B \subset X \times \Lambda$ is an isolating neighborhood for $F$, and $S=\operatorname{Inv}(B, F)$. From the point of view of the computational algorithms, it is more convenient to additionally assume the following condition similar in spirit to A1. As it is made clear via Remarks 4.3 and 5.2, these two assumptions are computationally compatible.

A1': For each grid element $Q \in \mathcal{Q}$ the set $\mathcal{B}_{Q}:=\mathcal{X}\left(\bigcup_{\lambda \in Q} B_{\lambda}\right)$ has the property that $S_{Q}=\operatorname{Inv}\left(\left|\mathcal{B}_{Q}\right| \times Q, F_{Q}\right)$.

Now for each $Q \in \mathcal{Q}$ we consider a multivalued map $\mathcal{F}_{Q}: \mathcal{B}_{Q} \rightrightarrows \mathcal{X}$ with the property that

$$
f(G, Q) \subset \operatorname{int}\left(\left|\mathcal{F}_{Q}(G)\right|\right)
$$

for all $G \in \mathcal{B}_{Q}$. Observe that if $\lambda \in Q$, then $\mathcal{F}_{Q}$ is an outer approximation of $f_{\lambda}$ restricted to $\left|\mathcal{B}_{Q}\right|$. We organize the collection of $\mathcal{F}_{Q}$ via the following definition. Set

$$
\mathcal{B}:=\bigcup_{Q \in \mathcal{Q}}\left(\mathcal{B}_{Q} \times\{Q\}\right) \subset \mathcal{X} \times \mathcal{Q}
$$

A combinatorialization of $F$ on $|\mathcal{B}|$ is the combinatorial multivalued map $\mathcal{F}: \mathcal{B} \rightrightarrows \mathcal{X} \times \mathcal{Q}$ defined by

$$
\mathcal{F}(G, Q)=\mathcal{F}_{Q}(G) \times\{Q\} .
$$

Note that $\mathcal{F}$ is composed of a collection of outer approximations of each $F_{Q}$.

The following proposition indicates how outer approximations are used to capture invariant sets.

Proposition 3.1. Suppose $\mathrm{A}^{\prime}$ holds and $\mathcal{F}$ is a combinatorialization of $F$ on $|\mathcal{B}|$. Let $Q \in \mathcal{Q}$, and suppose $\mathcal{Y} \subset \mathcal{B}_{Q}$. If $\mathcal{N}$ is the maximal subset of $\mathcal{Y}$ such that the restriction $\mathcal{F}_{Q}: \mathcal{N} \rightrightarrows \mathcal{N}$ is closed, then

$$
\operatorname{Inv}\left(|\mathcal{Y}| \times Q, F_{Q}\right)=\operatorname{Inv}\left(|\mathcal{N}| \times Q, F_{Q}\right) .
$$

Copyright (c) by SIAM. Unauthorized reproduction of this article is prohibited. 
Proof. Let $(x, \lambda) \in \operatorname{Inv}\left(|\mathcal{Y}| \times Q, F_{Q}\right)$, and choose any $G \in \mathcal{Y}$ that contains $x$. Let $\gamma_{x}$ be a complete orbit of $F_{Q}$ through $(x, \lambda)$ in $\operatorname{Inv}\left(|\mathcal{Y}| \times Q, F_{Q}\right)$. Since $\mathcal{F}_{Q}$ satisfies (3.1), there exists a sequence $\left\{G_{n}\right\}$ in $\mathcal{Y}$ with $G_{0}=G$ and $G_{n+1} \in \mathcal{F}_{Q}\left(G_{n}\right)$ such that $\gamma_{x}(n) \in G_{n} \times Q$ for all $n \in \mathbb{Z}$. Therefore, $G \in \mathcal{N}$, because it lies in a closed subgraph $\left\{G_{n}\right\}$ of $\mathcal{Y}$, which implies that $\left\{G_{n}\right\} \subset \mathcal{N}$, and thus $\gamma_{x} \subset|\mathcal{N}| \times Q$. Hence $(x, \lambda) \in \operatorname{Inv}\left(|\mathcal{N}| \times Q, F_{Q}\right)$. This implies that $\operatorname{Inv}\left(|\mathcal{Y}| \times Q, F_{Q}\right) \subset \operatorname{Inv}\left(|\mathcal{N}| \times Q, F_{Q}\right)$. Since the opposite inclusion is trivial, this concludes the proof.

Proposition 3.1 states that the maximal invariant set is captured by the largest closed subgraph of a combinatorialization. We have already commented that grids of arbitrarily small diameter exist in general. If $\mathcal{S}_{Q}$ is the largest closed subgraph of $\mathcal{B}_{Q}$, then it follows from the results in [9] that $\left|\mathcal{S}_{Q}\right|$ converges to $S_{Q}$ in Hausdorff metric as the grid diameters of $\mathcal{X}$ and $\mathcal{Q}$ tend to zero and the amount of overestimation in the computation of the outer approximation of the image of $f$ by $\mathcal{F}$ tends to zero; see Theorem 5.8 and Lemma 7.6 of [9]. We do not provide details here, because even though the convergence results prove that the maximal invariant set can be approximated arbitrarily closely if computations are performed on a sufficiently fine scale, in practice we fix a priori a finest resolution in the phase space and the parameter space with which we do our computations.

3.1. Constructing Conley-Morse graphs. Recall that we have assumed that $F$ satisfies A1. In addition, for the remainder of this section we assume that the set $B$ and the grid $\mathcal{X}$ are chosen in such a way that $\mathrm{A}^{\prime}$ is satisfied.

A natural starting point for examining the global structure of both a dynamical system and a directed graph is to look for recurrence. The recurrent set of $\mathcal{F}_{Q}: \mathcal{S}_{Q} \rightrightarrows \mathcal{X}$ is defined by

$$
\mathcal{R}_{Q}:=\left\{G \in \mathcal{S}_{Q} \mid \text { there exists a nontrivial path from } G \text { to } G \text { in } \mathcal{S}_{Q}\right\},
$$

where a nontrivial path is any path of nonzero length, including the case of a loop from a vertex to itself. The recurrent set $\mathcal{R}_{Q}$ is naturally partitioned into equivalence classes $\left\{\mathcal{M}_{Q}(p) \mid p \in \mathcal{P}_{Q}\right\}$ called combinatorial Morse sets according to the following equivalence relation:

$G \simeq H$ if and only if there exist a path in $\mathcal{F}_{Q}$ from $G$ to $H$ and a path in $\mathcal{F}_{Q}$ from $H$ to $G$.

Since every node in $\mathcal{S}_{Q}$ that lies on a cycle is an element of $\mathcal{R}_{Q}$, we can define a strict partial order on the indexing set $\mathcal{P}_{Q}$ by setting $p>_{Q} q$ if there exist $G \in \mathcal{M}_{Q}(p), H \in \mathcal{M}_{Q}(q)$, and a path from $G$ to $H$ in $\mathcal{F}_{Q}$.

Observe that this construction implies that a combinatorial Morse decomposition can be represented as a directed graph. Let $\operatorname{MG}\left(\mathcal{F}_{Q}\right)$ denote the acyclic directed graph with vertices consisting of the elements of $\mathcal{P}_{Q}$ and the minimal set of directed edges $p \rightarrow q$ which generate $p>_{Q} q$ under transitivity. The following proposition states that given a combinatorial Morse decomposition for an outer approximation $\mathcal{F}_{Q}$, there is a Morse decomposition of $F_{Q}$ such that $\operatorname{MG}\left(\mathcal{F}_{Q}\right)$ is the Morse graph over $Q$ for the Morse decomposition.

Proposition 3.2. Assume $\mathrm{A} 1$ and $\mathrm{A} 1^{\prime}$ are satisfied. Let $Q \in \mathcal{Q}$, and let $\left\{\mathcal{M}_{Q}(p) \mid p \in \mathcal{P}_{Q}\right\}$ be the set of combinatorial Morse sets for $\mathcal{F}_{Q}$. If $\mathcal{F}_{Q}\left(\mathcal{M}_{Q}(p)\right) \subset \mathcal{B}_{Q}$ for all $p \in \mathcal{P}_{Q}$, then the acyclic directed graph $\mathrm{MG}\left(\mathcal{F}_{Q}\right)$ which represents the combinatorial Morse sets is a Morse 
graph over $Q$ for the Morse decomposition of $S_{Q}$ defined by

$$
\mathbf{M}\left(S_{Q}\right):=\left\{\operatorname{Inv}\left(\left|\mathcal{M}_{Q}(p)\right| \times Q, F_{Q}\right) \mid p \in \mathcal{P}_{Q}\right\} .
$$

Moreover, each $\left|\mathcal{M}_{Q}(p)\right|$ is an isolating neighborhood for $\operatorname{Inv}\left|\mathcal{M}_{Q}(p)\right|$.

Proof. By [9, Theorem 4.1], we know that $\left|\mathcal{M}_{Q}(p)\right| \times Q$ is an isolating neighborhood for $F_{Q}$, and by [9, Corollary 4.2],

$$
\mathbf{M}\left(S_{Q}\right):=\left\{M_{Q}(p):=\operatorname{Inv}\left(\left|\mathcal{M}_{Q}(p)\right| \times Q, F_{Q}\right) \mid p \in\left(\mathcal{P}_{Q},>_{Q}\right)\right\}
$$

is a Morse decomposition of $S_{Q}$.

Remark 3.3. Observe that Proposition 3.2 implies that once an appropriate combinatorialization of $F$ has been computed, then for each $Q \in \mathcal{Q}$ a Morse graph $\operatorname{MG}\left(\mathcal{F}_{Q}\right)$ over $Q$ is determined which can be associated with a true Morse decomposition $\mathbf{M}\left(S_{Q}\right)$ for $F_{Q}$, and this in turn provides a Morse decomposition $\mathbf{M}\left(S_{\lambda}\right)$ for $f_{\lambda}$ for each $\lambda \in Q$.

The algorithms used to compute the Conley-Morse graphs are discussed in greater detail in section 4. For the moment we remark that we use the algorithm presented in section 2.2 of [1] to compute the Morse graph $\operatorname{MG}\left(\mathcal{F}_{Q}\right)$. There are a variety of algorithms for determining index pairs (see $[8,18,19,24]$ ), and in this paper we adopt the approach of [19]; see Remark 4.3. For systems defined in $\mathbb{R}^{n}$ and simplicial or rectangular grids, there exist algorithms to compute the induced map on homology $[8,14,20]$. Hence for each $Q \in \mathcal{Q}$ the Conley-Morse graph $\operatorname{CMG}\left(\mathcal{F}_{Q}\right)$ can be determined in a fairly general setting.

3.2. Comparing Conley-Morse graphs. We now turn to the question of comparing the dynamical information over different parameter regions via Conley-Morse graphs. Recall that we assume that the grid $\mathcal{Q}$ has the simple intersection property. Furthermore, we continue to assume that $\mathrm{A} 1$ and $\mathrm{A} 1^{\prime}$ are satisfied. We also assume that $\mathrm{CMG}\left(\mathcal{F}_{Q}\right)$ has been computed for each $Q \in \mathcal{Q}$. We begin with a few definitions.

Definition 3.4. To each $Q \in \mathcal{Q}$, there is an associated $\operatorname{CMG}\left(\mathcal{F}_{Q}\right)$. Consider $Q_{0}, Q_{1} \in \mathcal{Q}$ such that $Q_{0} \cap Q_{1} \neq \emptyset$. The clutching graph $\mathcal{J}\left(Q_{0}, Q_{1}\right)$ is defined to be the bipartite graph with vertices $\mathcal{P}_{Q_{0}} \cup \mathcal{P}_{Q_{1}}$ (the union of the vertices from $\operatorname{MG}\left(\mathcal{F}_{Q_{0}}\right)$ and $\operatorname{MG}\left(\mathcal{F}_{Q_{1}}\right)$ ) and with an edge $(p, q) \in \mathcal{P}_{Q_{0}} \times \mathcal{P}_{Q_{1}}$ if $\mathcal{M}_{Q_{0}}(p) \cap \mathcal{M}_{Q_{1}}(q) \neq \emptyset$.

Observe that if every vertex in $\mathcal{P}_{Q_{0}}$ in the clutching graph $\mathcal{J}\left(Q_{0}, Q_{1}\right)$ has a unique edge, then we can define the clutching function

$$
\iota_{Q_{1}, Q_{0}}: \mathcal{P}_{Q_{0}} \rightarrow \mathcal{P}_{Q_{1}}
$$

by $\iota_{Q_{1}, Q_{0}}(p):=q$ for each edge $(p, q)$ of $\mathcal{J}\left(Q_{0}, Q_{1}\right)$.

Definition 3.5. Consider the set of Conley-Morse graphs over the grid elements of the parameter space, i.e., $\left\{\operatorname{CMG}\left(\mathcal{F}_{Q}\right) \mid Q \in \mathcal{Q}\right\}$. Let $Q_{0}, Q_{1} \in \mathcal{Q}$ such that $Q_{0} \cap Q_{1} \neq \emptyset$. If the clutching function $\iota_{Q_{1}, Q_{0}}: \mathcal{P}_{Q_{0}} \rightarrow \mathcal{P}_{Q_{1}}$ is defined and gives a directed graph isomorphism from $\operatorname{MG}\left(\mathcal{F}_{Q_{0}}\right)$ to $\operatorname{MG}\left(\mathcal{F}_{Q_{1}}\right)$, then we say that the Conley-Morse graphs over $Q_{0}$ and $Q_{1}$, $\operatorname{CMG}\left(\mathcal{F}_{Q_{0}}\right)$ and $\operatorname{CMG}\left(\mathcal{F}_{Q_{1}}\right)$, are equivalent. The equivalence classes of $\left\{\operatorname{CMG}\left(\mathcal{F}_{Q}\right) \mid Q \in \mathcal{Q}\right\}$ with respect to the transitive closure of this relation are called continuation classes.

Remark 3.6. We require that $\iota_{Q_{1}, Q_{0}}$ generate a directed graph isomorphism as opposed to the weaker condition that $\iota_{Q_{1}, Q_{0}}$ be a bijection, because differences in the partial order may indicate a difference in the dynamics. 
Proposition 3.7. Assume $\mathrm{A} 1$ and $\mathrm{A}^{\prime}$ are satisfied. Let $Q_{0}, Q_{1} \in \mathcal{Q}$ such that $Q_{0} \cap Q_{1} \neq \emptyset$. If the clutching function $\iota_{Q_{1}, Q_{0}}: \mathcal{P}_{Q_{0}} \rightarrow \mathcal{P}_{Q_{1}}$ is a directed graph isomorphism, then there exists a Morse decomposition

$$
\mathbf{M}\left(S_{Q_{0} \cup Q_{1}}\right)=\left\{M_{Q_{0} \cup Q_{1}}(r) \mid r \in \mathcal{P}_{Q_{0} \cup Q_{1}}\right\}
$$

with admissible order $>_{Q_{0} \cup Q_{1}}$ such that its restriction is the same as the Morse decomposition $\mathbf{M}\left(S_{Q_{i}}\right)$ over $Q_{i}$ for each $i=0,1$. Specifically, there is a natural correspondence $\pi_{i}: \mathcal{P}_{Q_{0} \cup Q_{1}} \rightarrow \mathcal{P}_{Q_{i}}$ such that

$$
M_{Q_{i}}\left(\pi_{i}(r)\right)=M_{Q_{0} \cup Q_{1}}(r) \cap\left(X \times Q_{i}\right) \text { for any } r \in \mathcal{P}_{Q_{0} \cup Q_{1}},
$$

and $>_{Q_{0} \cup Q_{1}}$ agrees with $>_{Q_{i}}$ through the identification. Furthermore, the nonzero eigenvalues associated to the index maps for pairs of corresponding Morse sets $M_{Q_{0}}\left(\pi_{0}(r)\right)$ and $M_{Q_{1}}\left(\pi_{1}(r)\right)$ are the same.

Proof. Define

$$
\mathcal{P}_{Q_{0} \cup Q_{1}}=\left\{(p, q) \in \mathcal{P}_{Q_{0}} \times \mathcal{P}_{Q_{1}} \mid q=\iota_{Q_{1}, Q_{0}}(p)\right\},
$$

and the natural correspondence $\pi_{i}: \mathcal{P}_{Q_{0} \cup Q_{1}} \rightarrow \mathcal{P}_{Q_{i}}$. Then one can introduce a well-defined partial order $>_{Q_{0} \cup Q_{1}}$ on $\mathcal{P}_{Q_{0} \cup Q_{1}}$ from the isomorphic partial order $>_{Q_{i}}$ on $\mathcal{P}_{Q_{i}}$, and $\pi_{i}$ becomes an order-preserving isomorphism.

Now define

$$
M_{Q_{0} \cup Q_{1}}(r)=M_{Q_{0}}\left(\pi_{0}(r)\right) \cup M_{Q_{1}}\left(\pi_{1}(r)\right) .
$$

It follows from the construction that the collection $\left\{M_{Q_{0} \cup Q_{1}}(r) \mid r \in \mathcal{P}_{Q_{0} \cup Q_{1}}\right\}$ forms a Morse decomposition over $Q_{0} \cup Q_{1}$. The result now follows from the sheaf property of Morse decompositions and the continuation property of the Conley index.

Proposition 3.7 implies that if $\operatorname{CMG}\left(\mathcal{F}_{Q_{0}}\right)$ and $\operatorname{CMG}\left(\mathcal{F}_{Q_{1}}\right)$ belong to the same continuation class, then there is a path in the parameter space along which the underlying Morse decompositions are related by continuation, and the Conley indices of the corresponding Morse sets are isomorphic.

Remark 3.8. It is important to note that belonging to the same continuation class is a weak equivalence relation. In particular, it is possible that $\lambda_{0}, \lambda_{1} \in Q \in \mathcal{Q}$ and yet $S_{\lambda_{0}}$ and $S_{\lambda_{1}}$ are not topologically conjugate. Thus, membership in the same continuation class does not imply equivalence on the level of conjugacy. Heuristically, this is because we are studying the dynamics on the level of the grid elements, and differences in the dynamics that lie below this scale cannot be observed. Similarly, it is possible that $\operatorname{CMG}\left(\mathcal{F}_{Q_{0}}\right)$ and $\operatorname{CMG}\left(\mathcal{F}_{Q_{1}}\right)$ lie in different continuation classes, but the dynamics of $f_{\lambda_{0}}$ on $S_{Q_{0}}$ for $\lambda_{0} \in Q_{0}$ and $f_{\lambda_{1}}$ on $S_{Q_{1}}$ for $\lambda_{1} \in Q_{1}$ are topologically equivalent. To see that this is the case, consider $\lambda_{i}=\lambda \in Q_{0} \cap Q_{1}$, but $\operatorname{CMG}\left(\mathcal{F}_{Q_{0}}\right)$ and $\operatorname{CMG}\left(\mathcal{F}_{Q_{1}}\right)$ do not belong to the same continuation class. The heuristic explanation for this is that topological conjugacy is independent of the size of the dynamic structures, but the construction of $\mathcal{F}$ clearly depends on the size of the grid decomposition.

Finally, it is possible to construct examples in which $\operatorname{CMG}\left(\mathcal{F}_{Q_{0}}\right)$ and $\operatorname{CMG}\left(\mathcal{F}_{Q_{1}}\right)$ belong to the same continuation class and $Q_{0} \cap Q_{1} \neq \emptyset$, but $\iota_{Q_{1}, Q_{0}}$ is not a directed graph isomorphism, or even $\iota_{Q_{1}, Q_{0}}$ may not be defined. This situation can arise from a lack of resolution in either the phase space or parameter space. In fact, in practice (see section 4.6) we employ a 
local subdivision algorithm that in principle reduces - but does not necessarily eliminate - the occurrence of phenomena of this type.

The thesis of this paper is that, given grids for the phase space and parameter space, a useful database for the global dynamics is a list of the continuation classes and their relative connectivity. To be more precise, we introduce the following notion.

Definition 3.9. Assume $\mathrm{A} 1$ and $\mathrm{A}^{\prime}$ are satisfied. The associated continuation graph $\mathrm{CG}(\mathcal{F})$ is a graph whose vertices are the continuation classes

$$
\{(\operatorname{CMG}(j), \mathcal{Q}(j)) \mid j=1, \ldots, J\}
$$

where $\mathcal{Q}(k) \subset \mathcal{Q}$ is the set of parameter boxes associated with the $k$ th continuation class and $\operatorname{CMG}(k)=\operatorname{CMG}\left(\mathcal{F}_{Q}\right)$ for some $Q \in \mathcal{Q}(k)$. Note that all Conley-Morse graphs $\operatorname{CMG}\left(\mathcal{F}_{Q}\right)$ over $Q \in \mathcal{Q}(k)$ are isomorphic. There is an edge between the $j$ th and $k$ th vertices in $\operatorname{CG}(\mathcal{F})$ if there exist $Q \in \mathcal{Q}(j)$ and $Q^{\prime} \in \mathcal{Q}(k)$ such that $Q \cap Q^{\prime} \neq \emptyset$.

The continuation graph is our database. Of course, additional, problem specific information can also be stored. However, as will be indicated in the context of the density-dependent Leslie model, this database provides an extremely compressed yet useful means of describing the global dynamics over a broad range of parameter values. We return to these issues when we use the database to investigate the Leslie model.

4. Building the database using rectangular grids. The results of section 3 indicate how a combinatorialization of $F$ leads to a database. In this section we describe how a combinatorialization can be effectively computed, including details of selected computational aspects of the method.

The optimal choice of a suitable grid is determined by the structure of $X, \Lambda$, and $B \subset$ $X \times \Lambda$. For many applications $\Lambda \subset \mathbb{R}^{m}$ is a rectangular region, $X=\mathbb{R}^{n}$, and $B$ is also a rectangular region; that is, $B=R \times \Lambda$ for some rectangular region $R \subset \mathbb{R}^{n}$. For convenience, we make this assumption, i.e., $B_{\lambda}=R$ for all $\lambda \in \Lambda$, throughout the rest of this paper. This leads to the use of a pair of rectangular grids

$$
\mathcal{Q}=\left\{\prod_{i=1}^{m}\left[b_{i}+q_{i} \frac{\zeta_{i}}{K_{i}}, b_{i}+\left(q_{i}+1\right) \frac{\zeta_{i}}{K_{i}}\right] \mid q_{j} \in\left\{0, \ldots, K_{j}-1\right\}, j=1, \ldots, m\right\},
$$

where $b, \zeta \in \mathbb{R}^{m}$ and $K \in \mathbb{Z}^{m}$,

$$
\mathcal{X}^{(d)}=\left\{\prod_{i=1}^{n}\left[a_{i}+k_{i} \frac{\xi_{i}}{2^{d}}, a_{i}+\left(k_{i}+1\right) \frac{\xi_{i}}{2^{d}}\right] \mid k_{j} \in \mathbb{Z}, j=1, \ldots, n\right\},
$$

where $d \in \mathbb{Z}^{+}$and $a, \xi \in \mathbb{R}^{n}$, and

$$
\mathcal{B}^{(d)}:=\mathcal{X}^{(d)}(R) \subset \mathcal{X}^{(d)} .
$$

The choices of $K$ and $d$ define the accuracy of computations, and the choices of $a, b, \xi$, and $\zeta$ are determined by the regions one wishes to study. The parameter $d$ allows for the use of an iterative multiscale method described later that is essential for efficient computation of the dynamics.

Copyright (c) by SIAM. Unauthorized reproduction of this article is prohibited. 
4.1. Constructing an outer approximation. For each $Q \in \mathcal{Q}$ we construct an outer approximation $\mathcal{F}_{Q}^{(d)}: \mathcal{B}^{(d)} \rightrightarrows \mathcal{X}^{(d)}$ as follows. For each grid element $G \in \mathcal{B}^{(d)}$, interval arithmetic [16] is used to compute a rectangular box $\beta(G)$ which contains the image $f(G, Q)$. More precisely, the edges of the rectangular grid element $G \times Q$ (which are intervals) are inserted directly into the formula for $f$ in place of corresponding variables, and the value of $f(G, Q)$ is evaluated using interval arithmetic which provides a rigorous result in the form of a product of intervals. For each $G \in \mathcal{B}^{(d)}$ define

$$
\mathcal{F}_{Q}^{(d)}(G):=\left\{H \in \mathcal{X}^{(d)} \mid H \cap \beta(G) \neq \emptyset\right\}
$$

and observe that $f(G, Q) \subset \operatorname{int}\left|\mathcal{F}_{Q}^{(d)}(G)\right|$; see Figure 1. Thus, $\mathcal{F}_{Q}^{(d)}$ is an outer approximation as defined in (3.1).

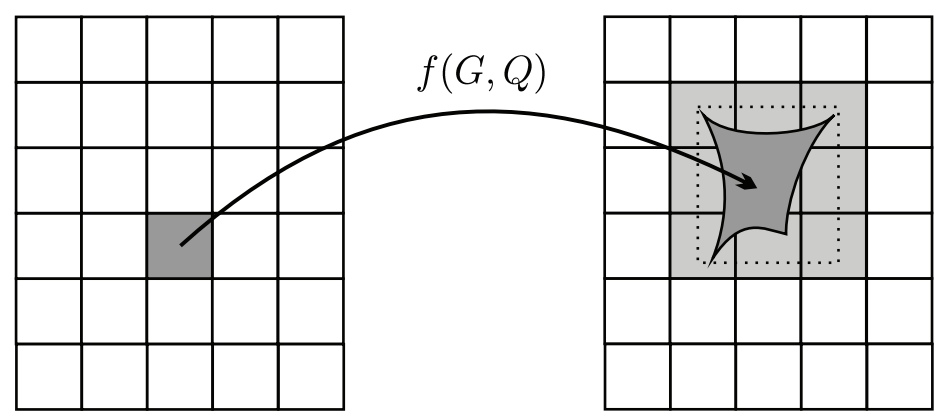

Figure 1. A rigorous enclosure of the image of each grid box (dark gray) is computed with interval arithmetic (indicated by the dotted line) and then covered by grid boxes (light gray).

Having described the construction of $\mathcal{F}_{Q}^{(d)}: \mathcal{B}^{(d)} \rightrightarrows \mathcal{X}^{(d)}$, we turn to the need for a multiscale approach. Observe that the number of grid elements in $\mathcal{B}^{(d)}$ is $2^{\text {nd }}$, and hence the size of the graph of $\mathcal{F}_{Q}$ grows exponentially as a function either of discretization size or of dimension of the problem. However, we are interested only in the largest closed subgraph of $\mathcal{F}_{Q}$ on $\mathcal{B}^{(d)}$, and thus we use only the restriction of $\mathcal{F}_{Q}$ to $\mathcal{S}_{Q}^{(d)}$, where $\left|\mathcal{S}_{Q}^{(d)}\right| \times Q$ covers $\operatorname{Inv} B_{Q}$, which is often a small subset of $B_{Q}$.

4.2. Iterative multiscale identification of the invariant set. To avoid unnecessary computation, we use the following iterative multiscale approach motivated by $[6,7]$. Let $d_{0}, d_{1}, \ldots, d_{\ell}$ be an increasing sequence of positive integers. Fix $Q \in \mathcal{Q}$. Construct $\mathcal{F}_{Q}^{\left(d_{0}\right)}: \mathcal{B}^{\left(d_{0}\right)} \rightrightarrows \mathcal{X}^{\left(d_{0}\right)}$. Following the algorithms in [1], we compute the maximal closed directed graph $\mathcal{S}_{Q}^{\left(d_{0}\right)}$ in $\mathcal{F}_{Q}^{\left(d_{0}\right)}$. Define $\mathcal{Y}^{\left(d_{1}\right)} \subset \mathcal{B}^{\left(d_{1}\right)}$ to be the grid for $\left|\mathcal{S}_{Q}^{\left(d_{0}\right)}\right|$. Construct $\mathcal{F}_{Q}^{\left(d_{1}\right)}: \mathcal{Y}^{\left(d_{1}\right)} \rightrightarrows \mathcal{X}^{\left(d_{1}\right)}$. We repeat the construction of $\mathcal{S}_{Q}^{\left(d_{i}\right)}$ and $\mathcal{F}_{Q}^{\left(d_{i}\right)}: \mathcal{Y}^{\left(d_{i}\right)} \rightrightarrows \mathcal{X}^{\left(d_{i}\right)}$ until $i=l$. Note that since we assume $\mathrm{A} 1^{\prime}$, Proposition 3.1 implies that $\operatorname{Inv}\left(\left|\mathcal{S}^{\left(d_{\ell}\right)}\right| \times Q\right)=\operatorname{Inv}\left(\left|\mathcal{B}_{Q}^{\left(d_{0}\right)}\right| \times Q\right)$.

Example 4.1. To put the above construction into perspective, consider the Leslie model (1.3), where we fix $p=0.7$. Set

$$
\begin{aligned}
& \Lambda:=\left\{\left(\theta_{1}, \theta_{2}\right) \in[8,37] \times[3,50]\right\}, \\
& R:=[-0.001,320.056] \times[-0.001,224.040] \text { for all } \lambda \in \Lambda .
\end{aligned}
$$

Copyright (C) by SIAM. Unauthorized reproduction of this article is prohibited. 

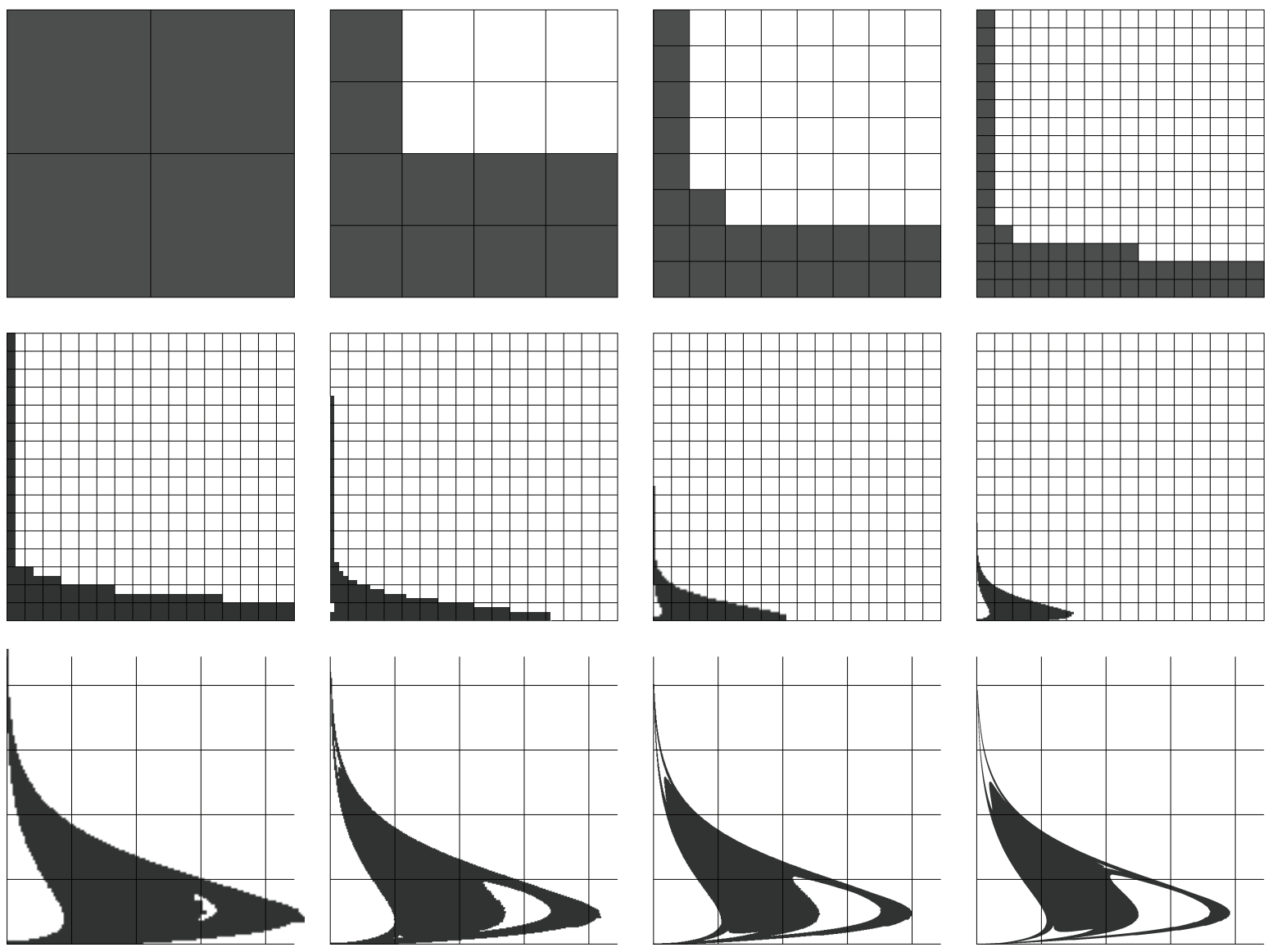

Figure 2. Computation of $\mathcal{S}_{Q}^{\left(d_{i}\right)}$ by means of gradual refinements. In the first row, the lines indicate the subdivision of the region $X$ into $4^{n}$ boxes for $n=1, \ldots, 4$. No more lines are added in the second row to keep the picture clear. The third row shows a magnified fragment of the region $\mathcal{S}_{Q}^{\left(d_{i}\right)}$ with the lines corresponding to those in the second row. Parameter box $Q$ coordinates: $\left(q_{1}, q_{2}\right)=(20,33)$.

The grid $\mathcal{Q}$ in $\Lambda$ is given by (4.1) with $m=2, b_{1}=8, b_{2}=3, \zeta_{1}=29, \zeta_{2}=47$, and $K_{1}=K_{2}=50$, while the grids $\mathcal{X}^{(d)}$ in $X=\mathbb{R}^{2}$ are given for $d=1, \ldots, 12$ by (4.2) with $n=2$, $a_{1}=a_{2}=-0.001, \xi_{1}=320.057$, and $\xi_{2}=224.041$. Figure 2 indicates the evolution of the sets $\mathcal{S}_{Q}^{(d)}$ for $d=1, \ldots, 12$, where $Q \in \mathcal{Q}$ is given by $\left(q_{1}, q_{2}\right)=(20,33)$ in (4.1).

Remark 4.2. Observe that the diameter of $\left|\mathcal{F}_{Q}^{(d)}(G)\right|$ depends on the diameter of $\beta(G)$, which in turn is dependent both on the diameter of $G$ and $Q$. Currently, we fix the grid $\mathcal{Q}$ and then iteratively refine $\mathcal{X}^{(d)}$. Thus, the size of $Q \in \mathcal{Q}$ puts an upper bound on the number of iterative steps that lead to a useful refinement of the outer approximation $\mathcal{F}_{Q}^{(d)}: \mathcal{Y}^{(d)} \rightrightarrows \mathcal{X}^{(d)}$. A more sophisticated approach would involve an iterative method for both the phase space and the parameter space.

Remark 4.3. By Proposition 3.1, $S_{Q} \subset\left|\mathcal{S}_{Q}^{\left(d_{i}\right)}\right| \times Q$ for $i=0, \ldots, l$. Moreover, since $\mathcal{S}_{Q}^{\left(d_{i}\right)}$ is the maximal closed subgraph, and combinatorial Morse sets are closed subgraphs, each combinatorial Morse set $\mathcal{M}_{Q}(p)$ for $p \in \mathcal{P}_{Q}$ is contained in $\mathcal{S}_{Q}^{\left(d_{i}\right)}$ for each $i=0, \ldots, \ell$. If 
$\mathcal{F}\left(\mathcal{M}_{Q}(p)\right) \subset \mathcal{B}_{Q}$ for all $p \in \mathcal{P}_{Q}$, then by Proposition 3.2, the neighborhoods $\left|\mathcal{M}_{Q}(p)\right|$ isolate Morse sets in a Morse decomposition of $S_{Q}$. Moreover, since Morse sets are recurrent, the condition $\mathcal{F}\left(\mathcal{M}_{Q}(p)\right) \subset \mathcal{B}_{Q}$ implies that

$$
\mathcal{F}_{Q}\left(\mathcal{F}_{Q}\left(\mathcal{M}_{Q}(p)\right) \backslash \mathcal{M}_{Q}(p)\right) \cap \mathcal{M}_{Q}(p)=\emptyset,
$$

where $\mathcal{F}_{Q}: \mathcal{X} \rightarrow \mathcal{X}$ is an outer approximation. As described in the next section, this allows us to readily compute the Conley index of $\operatorname{Inv}\left(\left|\mathcal{M}_{Q}\right|\right)$. If the condition $\mathcal{F}\left(\mathcal{M}_{Q}(p)\right) \subset \mathcal{B}_{Q}$ is not satisfied, then we cannot compute the index without extending our computation of $\mathcal{F}_{Q}$ outside of $\mathcal{B}_{Q}$, and this situation is reported in the output of our computations.

4.3. Conley-Morse graph computation. Given $\mathcal{F}_{Q}^{\left(d_{\ell}\right)}: \mathcal{S}_{Q}^{\left(d_{\ell}\right)} \rightrightarrows \mathcal{X}^{\left(d_{\ell}\right)}$ the algorithm in $\left[1\right.$, section 2.2] is used to compute the Morse graph $\operatorname{MG}\left(\mathcal{F}_{Q}\right)$, where $\mathcal{F}_{Q}:=\mathcal{F}_{Q}^{\left(d_{\ell}\right)}$. To produce the Conley-Morse graph over $Q$ requires the computation of the Conley indices of each Morse set. This is done using the algorithms in [14, 20] and the easy observation that if $\mathcal{M}_{Q}(p)$ is a combinatorial Morse set for $\mathcal{F}_{Q}$ and the condition (4.3) is satisfied for $\mathcal{F}_{Q}: \mathcal{M}_{Q}(p) \cup \mathcal{F}_{Q}\left(\mathcal{M}_{Q}(p)\right) \rightrightarrows \mathcal{X}$, then $\left(\mathcal{M}_{Q}(p) \cup \mathcal{F}_{Q}\left(\mathcal{M}_{Q}(p)\right), \mathcal{F}_{Q}\left(\mathcal{M}_{Q}(p)\right) \backslash \mathcal{M}_{Q}(p)\right)$ is a combinatorial index pair as in $[19,20]$. In practice, there are memory constraints associated with these algorithms for computing the Conley index. In the computations performed for this paper we did not attempt to compute the Conley index if the index pairs generated contained more than 400,000 boxes.

Example 4.4. Returning to Example 4.1, the computed Conley-Morse graph over $Q$ is indicated in Figure 3(a). The three Morse sets $M_{Q}\left(p_{i}\right), i=2,4,5$, indicated by the shaded boxes have no nonzero eigenvalues. The numbers in parentheses indicate the number of boxes that define each combinatorial Morse set $\mathcal{M}_{Q}\left(p_{i}\right)$. Remark 2.3.2 raises the possibilitybut does not imply - that $M_{Q}\left(p_{i}\right)=\emptyset$ for $i=2,4,5$. This possibility is reinforced by the observation that there are very few boxes in $\mathcal{M}_{Q}\left(p_{i}\right)$ for $i=2,4,5$.

To test whether the Morse sets with trivial index may, in fact, be numerical artifacts, we need to be able to study them at a finer level of resolution.

4.4. Combinatorial Morse set refinement. Observe that if $\mathcal{M}_{Q}(p)$ is a combinatorial Morse set determined by an outer approximation $\mathcal{F}_{Q}^{(d)}$, then the restriction $\mathcal{F}_{Q}^{(d)}: \mathcal{M}_{Q}(p) \rightrightarrows$ $\mathcal{M}_{Q}(p)$ is a closed graph. Choose $d^{\prime}$ greater than $d$. Define $\mathcal{Y}_{Q}^{\left(d^{\prime}\right)}$ to be the grid for $\left|\mathcal{M}_{Q}(p)\right|$, construct $\mathcal{F}_{Q}^{\left(d^{\prime}\right)}: \mathcal{Y}_{Q}^{\left(d^{\prime}\right)} \rightrightarrows \mathcal{Y}_{Q}^{\left(d^{\prime}\right)}$, and compute $\mathcal{S}_{Q}^{\left(d^{\prime}\right)}$, the maximal closed directed graph in $\mathcal{F}_{Q}^{\left(d^{\prime}\right)}$. By Proposition 3.1 all the recurrent dynamics of $\mathcal{M}_{Q}(p)$ is contained in $\mathcal{S}_{Q}^{\left(d^{\prime}\right)}$, because

$$
M_{Q}(p)=\operatorname{Inv}\left(\left|\mathcal{S}_{Q}^{\left(d^{\prime}\right)}\right|, F_{Q}\right)=\operatorname{Inv}\left(\left|\mathcal{M}_{Q}(p)\right|, F_{Q}\right) .
$$

4.5. Conley-Morse graph reduction test. The first step is to apply the Combinatorial Morse Set Refinement algorithm with $d=d_{\ell}$ and $d^{\prime}=d_{\ell}+1$. There are two possible outcomes.

1. $\mathcal{S}_{Q}^{\left(d^{\prime}\right)}=\emptyset$. In this case $M_{Q}(p)=\emptyset$, so we remove the vertex $p$ and replace $\operatorname{CMG}\left(\mathcal{F}_{Q}^{\left(d_{\ell}\right)}\right)$ with its trivial reduction.

2. $\mathcal{S}_{Q}^{\left(d^{\prime}\right)} \neq \emptyset$. In this case there are two other options: Either we halt and accept $\operatorname{CMG}\left(\mathcal{F}_{Q}^{\left(d_{\ell}\right)}\right)$ as the appropriate Conley-Morse graph over $Q$, or we apply the Combinatorial Morse Set Refinement procedure to $\mathcal{S}_{Q}^{\left(d^{\prime}\right)}$ and repeat the process.

Copyright ( $\odot$ by SIAM. Unauthorized reproduction of this article is prohibited. 


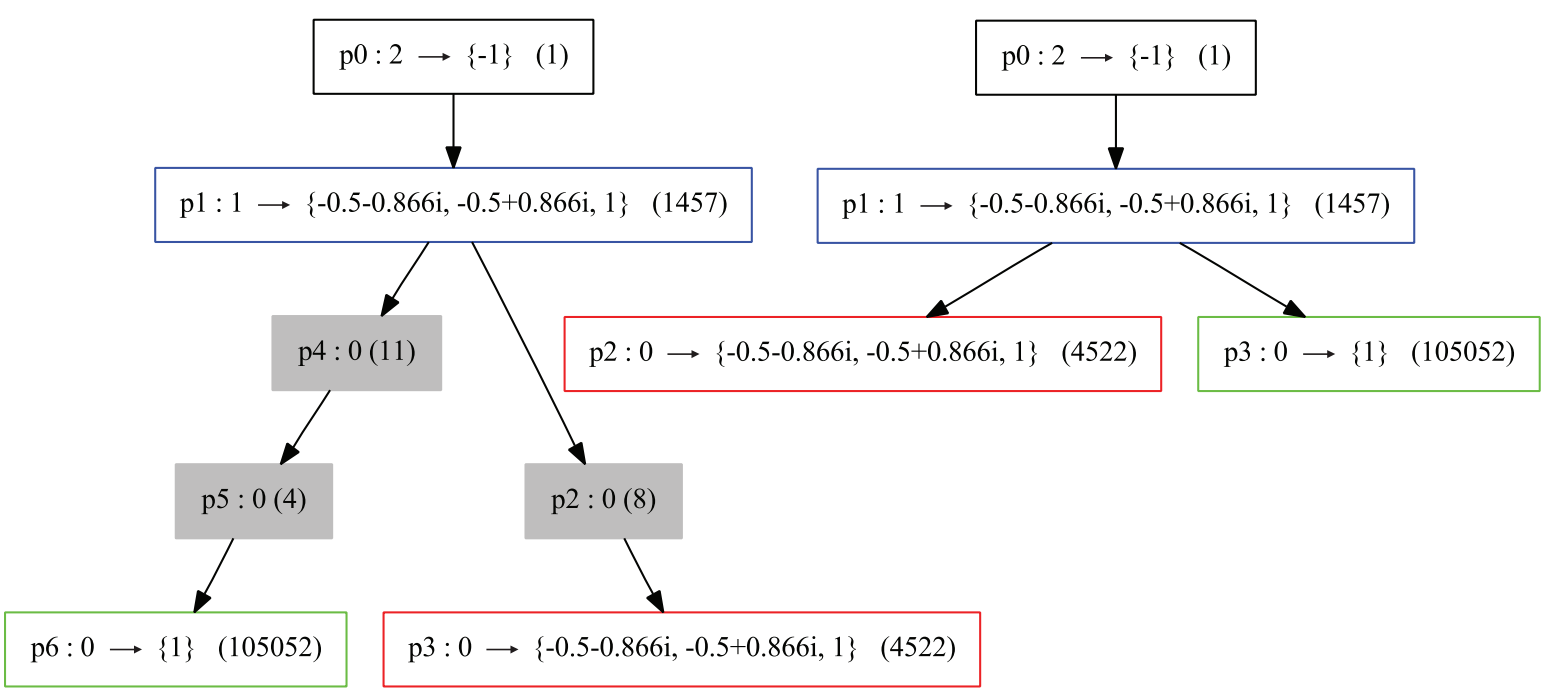

(a)

(b)

Figure 3. (a) The Conley-Morse graph computed over the parameter box $Q$ defined by $\left(q_{1}, q_{2}\right)=(20,33)$ and $d_{\ell}=12$. The three Morse sets indicated by the shaded boxes have no nonzero eigenvalues. (b) The trivially reduced Conley-Morse graph over $Q$.

In practice there are two constraints to this procedure. The first is the number of iterations before accepting during which a Morse set with trivial index remains in the Conley-Morse graph. In the computations performed for this paper we stopped after three iterations. The second arises from memory considerations. If the combinatorial Morse set consists of a large number of boxes, then representing it on a finer grid can produce an impractically large set. In the computations performed for this paper we did not apply the Combinatorial Morse Set Refinement to sets consisting of more than 40,000 boxes.

Example 4.5. Continuing with Example 4.4, after applying one iteration of the ConleyMorse Graph Reduction Test with $d^{\prime}=13$ it is determined that $M_{Q}\left(p_{k}\right)=\emptyset$ for $k=2,4,5$. This leads to the trivially reduced Conley-Morse graph indicated in Figure 3(b). The sets $\mathcal{M}_{Q}\left(p_{k}\right)$ for the reduced Conley-Morse graph over $Q$ are indicated in Figure 4.

Recall that the desired database is a continuation graph. The first step is to create the continuation classes. Observe that over each $Q \in \mathcal{Q}$ we have computed the trivially reduced Conley-Morse graphs $\operatorname{CMG}\left(\mathcal{F}_{Q}\right)$. We still retain the information of the boxes $\left\{\mathcal{M}_{Q}(p) \mid\right.$ $\left.p \in \mathcal{P}_{Q}\right\}$ which define the vertices of $\operatorname{CMG}\left(\mathcal{F}_{Q}\right)$ and of course the ordering $>_{Q}$ between the vertices.

4.6. Continuation graph construction. Let $Q_{0}$ and $Q_{1}$ be grid elements such that $Q_{0} \cap$ $Q_{1} \neq \emptyset$. In order to determine whether $\operatorname{CMG}\left(\mathcal{F}_{Q_{0}}\right)$ and $\operatorname{CMG}\left(\mathcal{F}_{Q_{1}}\right)$ are equivalent, we proceed with the following steps:

1. If the cardinalities of $\mathcal{P}_{Q_{0}}$ and $\mathcal{P}_{Q_{1}}$ differ, then $Q_{0}$ and $Q_{1}$ do not belong to the same continuation class.

2. If the cardinalities of $\mathcal{P}_{Q_{0}}$ and $\mathcal{P}_{Q_{1}}$ agree, then we construct the clutching graph (see comment preceding Definition 3.5). If the clutching graph defines a directed graph iso- 

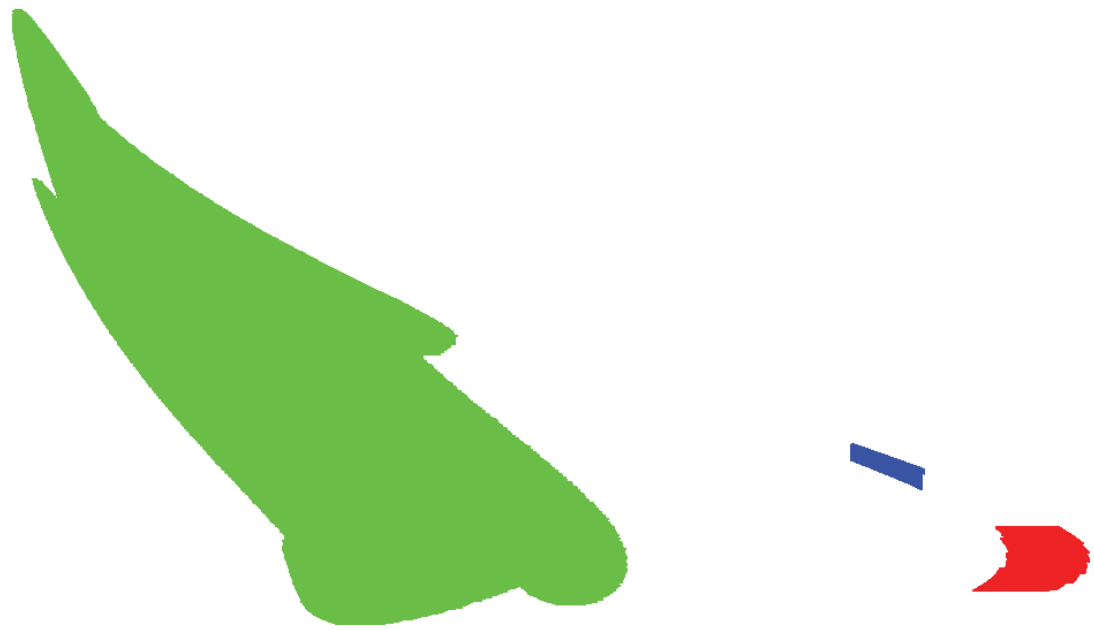

Figure 4. The sets $\mathcal{M}_{Q}\left(p_{i}\right), i=0,1,2,3$, for the reduced Conley-Morse graph from Figure 3(b). The color coding in the two figures matches; thus the green and red regions indicate attracting neighborhoods for $f_{\lambda}$ for all $\lambda$ in the parameter box $Q$ defined by $\left(q_{1}, q_{2}\right)=(20,33)$ and $d_{\ell}=12$. The set $\mathcal{M}_{Q}\left(p_{0}\right)$ covers the origin and consists of a single box at the lower left corner of the picture and is barely visible at this resolution.

morphism between the Morse graphs $\operatorname{MG}\left(\mathcal{F}_{Q_{0}}\right)$ and $\operatorname{MG}\left(\mathcal{F}_{Q_{1}}\right)$, then the Conley-Morse graphs $\operatorname{CMG}\left(\mathcal{F}_{Q_{0}}\right)$ and $\operatorname{CMG}\left(\mathcal{F}_{Q_{1}}\right)$ over $Q_{0}$ and $Q_{1}$ belong to the same continuation class.

3. The clutching graph can fail to define a directed graph isomorphism between the Morse graphs $\operatorname{MG}\left(\mathcal{F}_{Q_{0}}\right)$ and $\operatorname{MG}\left(\mathcal{F}_{Q_{1}}\right)$ in several ways:

- The partial orders do not agree, in which case $\operatorname{CMG}\left(\mathcal{F}_{Q_{0}}\right)$ and $\operatorname{CMG}\left(\mathcal{F}_{Q_{1}}\right)$ are not identified as belonging to the same continuation class.

- There is a vertex with no edge in the clutching graph, in which case $\operatorname{CMG}\left(\mathcal{F}_{Q_{0}}\right)$ and $\operatorname{CMG}\left(\mathcal{F}_{Q_{1}}\right)$ do not belong to the same continuation class.

- There is a vertex with two or more edges in the clutching graph. Recall that the edge $(p, q)$ is in the clutching graph if $\mathcal{M}_{Q_{0}}(p) \cap \mathcal{M}_{Q_{1}}(q) \neq \emptyset$. Assume the edges at the vertex $p$ are given by $\left\{\left(p, q_{i}\right) \mid i=1, \ldots, I\right\}$. Then we apply several iterations of the Combinatorial Morse Set Refinement algorithm to $\mathcal{M}_{Q_{0}}(p)$ and $\mathcal{M}_{Q_{1}}\left(q_{i}\right), i=1, \ldots, I$. If, after performing this at each vertex for which there 
are multiple edges, the clutching graph reduces to a directed graph isomorphism, then $\operatorname{CMG}\left(\mathcal{F}_{Q_{0}}\right)$ and $\operatorname{CMG}\left(\mathcal{F}_{Q_{1}}\right)$ belong to the same continuation class; otherwise $\operatorname{CMG}\left(\mathcal{F}_{Q_{0}}\right)$ and $\operatorname{CMG}\left(\mathcal{F}_{Q_{1}}\right)$ belong to different continuation classes.

This procedure applied to all pairs of adjacent boxes in the parameter space results in the set of continuation classes and the continuation graph which defines the database having been determined. Note that although this procedure admits the situation in which two Conley-Morse graphs are identified as belonging to the same continuation class even if the clutching function does not define a directed graph isomorphism, in such a case the conclusion of Proposition 3.7 still holds true, because the clutching function of the refined combinatorial Morse decompositions does provide the isomorphism.

5. Database for the Leslie model with $p=0.7$. In this section we present the results of the computational procedure described in section 4 applied to the density-dependent Leslie model (1.3) when $p=0.7$. The procedure was implemented in an efficient program written in $\mathrm{C}++$. An interactive presentation of the results of computations which we did can be found at http://chomp.rutgers.edu/database/, and the source code of the general purpose software used to compute this database has also been made freely available.

Observe that fixing $p=0.7$ results in a two-dimensional parameter space. We compute the continuation graph over the parameter space

$$
\Lambda:=\left\{\left(\theta_{1}, \theta_{2}\right) \in[8,37] \times[3,50]\right\} .
$$

We choose an equipartitioned $50 \times 50$ grid for this parameter space given by (4.1) with $m=2$, $b_{1}=8, b_{2}=3, \zeta_{1}=29, \zeta_{2}=47$, and $K_{1}=K_{2}=50$.

Recasting the Leslie model in the general form of (1.2) and recalling that $x_{1}$ and $x_{2}$ represent population sizes, we are interested in studying $F:\left(\mathbb{R}^{+}\right)^{2} \times \Lambda \rightarrow\left(\mathbb{R}^{+}\right)^{2} \times \Lambda$, where $\mathbb{R}^{+}:=[0, \infty)$.

There are two essential observations.

Remark 5.1. For $\lambda=\left(\theta_{1}, \theta_{2}\right)$, define

$$
B_{\lambda}:=\left\{\left(x_{1}, x_{2}\right) \in\left(\mathbb{R}^{+}\right)^{2} \mid 0 \leq x_{1} \leq 10\left(\theta_{1}+\theta_{2}\right) e^{-1}, 0 \leq x_{2} \leq 10 p\left(\theta_{1}+\theta_{2}\right) e^{-1}\right\} .
$$

A direct calculation shows that $F^{2}\left(\left(\mathbb{R}^{+}\right)^{2} \times \Lambda\right) \subset B:=\bigcup_{\lambda \in \Lambda} B_{\lambda} \times\{\lambda\}$. In particular, any invariant set for the Leslie model on $\left(\mathbb{R}^{+}\right)^{2}$ must lie in $B$. Furthermore, $F(0, \lambda)=(0, \lambda)$ and $\{0\} \times(0, \infty) \not \subset f_{\lambda}\left(\left(\mathbb{R}^{+}\right)^{2}\right)$.

Remark 5.2. There are some technical reasons why we do not compute on the set $B \subset$ $\left(\mathbb{R}^{+}\right)^{2} \times \Lambda$ as defined in Remark 5.1. First, $F(0, \lambda)=(0, \lambda)$ so that $B$ is not an isolating neighborhood as a subset of $\mathbb{R}^{2} \times \Lambda$. While this would not violate A1 restricted to $\left(\mathbb{R}^{+}\right)^{2} \times \Lambda$, it does imply that the Conley index of the invariant set $\{0\}$ computed restricted to $B$ would not be capable of measuring the hyperbolicity of the origin. Our approach to this problem is pragmatic; we can explicitly compute the eigenvalues at the origin as

$$
\mu_{ \pm}=\frac{\theta_{1} \pm \sqrt{\theta_{1}^{2}+4 p \theta_{2}}}{2} .
$$

Observe that restricted to $\Lambda, \mu_{+}>1$. This information can then be used to compute the index analytically. However, this is not the only reason not to compute on $B$. 
As exhibited in the figures in the next section, the maximal invariant set in $B$ comes very close to the coordinate axes, even though it does not touch the axes by Remark 5.1. This causes a problem again in isolating and computing the Conley index, even for some Morse sets that do not include the origin, when the computations are restricted to the first quadrant.

Given these issues, we have chosen to compute on a larger rectangle

$$
R:=[-0.001,320.056] \times[-0.001,224.040]
$$

in $\mathbb{R}^{2}$ and consider the origin analytically. With respect to the dynamics on $\mathbb{R}^{2}$, the origin undergoes a period-doubling bifurcation since $\mu_{-}<-1$ if $\theta_{2}>\frac{1}{p} \theta_{1}+1$ and $-1<\mu_{-}<0$ if $\theta_{2}<\frac{1}{p} \theta_{1}+1$. The eigenvector $v_{+}$associated with $\mu_{+}$can be chosen to lie in the positive orthant. The eigenvector associated with $\mu_{-}$lies outside the positive cone. Thus, this perioddoubling bifurcation has no direct impact on the ordering of the Morse decomposition of $S_{\lambda}:=\operatorname{Inv}\left(R, f_{\lambda}\right)$. Combining this with the observation that $\mu_{+}>1$, one can conclude that for any $\lambda \in \Lambda$ the origin is always a repeller for $S_{\lambda}$.

These observations imply that, even though the condition $\mathrm{A} 1^{\prime}$ does not hold near the period-doubling bifurcation, this bifurcation plays no role in the construction of the continuation classes. Moreover, the Conley index of the origin provides no information and hence can be ignored in the database.

Using the grids defined in Example 4.1, we compute the continuation graph using the iterative method in section 4.2 on the grids $\mathcal{B}^{(d)}$ for $d=6, \ldots, 12$. The output is indicated in Figure 5. Since there is no natural order on the continuation classes, we have labeled them from "Class 1" to "Class 17," according to their volume in parameter space, beginning with the largest region.

The advantage of the continuation graph is that it is dimension-independent, and thus it can be used to organize the information about the dynamics independent of the dimension of the parameter space. In this example, however, the parameter space is two-dimensional; thus we also present a continuation diagram in Figure 6 where each continuation class with more than one parameter box is identified by some color. Thus, it is easy to see the sets $\mathcal{Q}(j) \subset \mathcal{Q}$ which define the parameter values for which the Conley-Morse graphs are valid.

The Conley-Morse graphs associated to the continuation classes, which are the basic items of the database, are shown in the next subsection.

5.1. Catalogue of continuation classes. This section contains a list of Conley-Morse graphs and Morse decompositions at selected parameter boxes for all the continuation classes found for the two-dimensional Leslie model with two varying parameters, as discussed above.

Recall that each node in a Conley-Morse graph is labeled

$$
\mathrm{p} k: n \rightarrow\{*\},
$$

where $k$ labels the Morse set, $\{*\}$ indicates the nonzero eigenvalues, and $n$ indicates the level of homology of the index map on which these eigenvalues arise. If the $k$ th Morse set has no nonzero eigenvalues, then we write

$$
\mathrm{p} k: 0 \text {. }
$$

Copyright $\odot$ by SIAM. Unauthorized reproduction of this article is prohibited. 


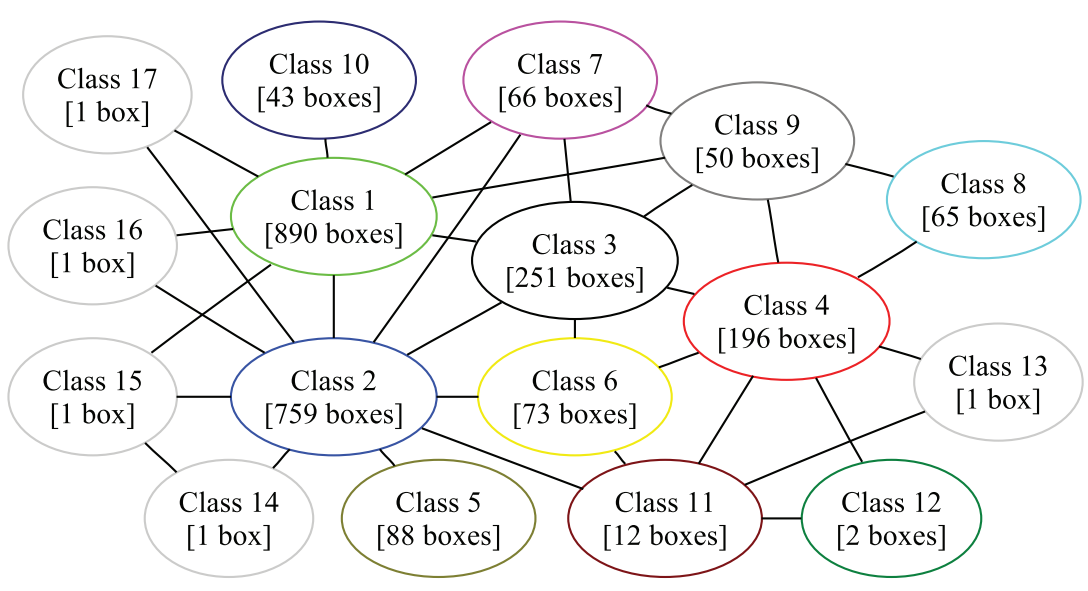

Figure 5. The continuation graph computed for the density-dependent Leslie population model (1.3) with $\Lambda=\left\{\theta=\left(\theta_{1}, \theta_{2}\right) \in[8,37] \times[3,50]\right\}$ and $p=0.7$. The label of each node indicates the class number and the number of boxes in $\mathcal{Q}(j)$, and the border color agrees with Figure 6.

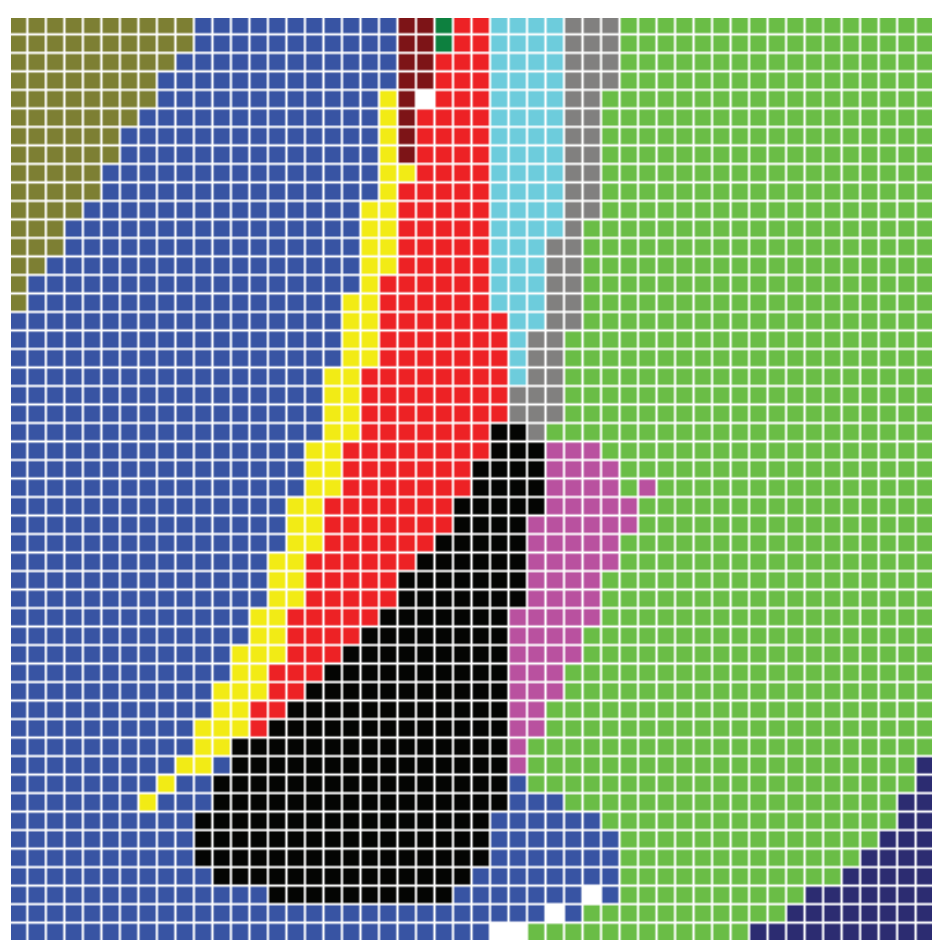

Figure 6. Continuation diagram computed for the two-dimensional Leslie population model with $\theta_{1} \in[8,37]$, $\theta_{2} \in[3,50], p=0.7$. Grid sizes: $50 \times 50$ in the parameter space for $\left(\theta_{1}, \theta_{2}\right)$ and $4096 \times 4096$ in the phase space $[-0.001,320.056] \times[-0.001,224.040]$.

Since, as indicated in Remark 5.2, the origin is an exceptional Morse set, we indicate it with a shaded box and do not include any index information. The boxes are color-coded to match the combinatorial Morse sets that are shown in the right panel. 
We do not consider the figures showing the combinatorial Morse sets to be part of the database for the following reasons:

1. The boxes that make up the combinatorial Morse sets will, in general, be different for each $Q \in \mathcal{Q}$.

2. For higher dimensional problems, visualizing the combinatorial Morse sets becomes impractical if not impossible.

3. Storing the combinatorial Morse sets becomes prohibitive for higher dimensional problems.

4. We do not have a method for directly querying the combinatorial Morse sets.

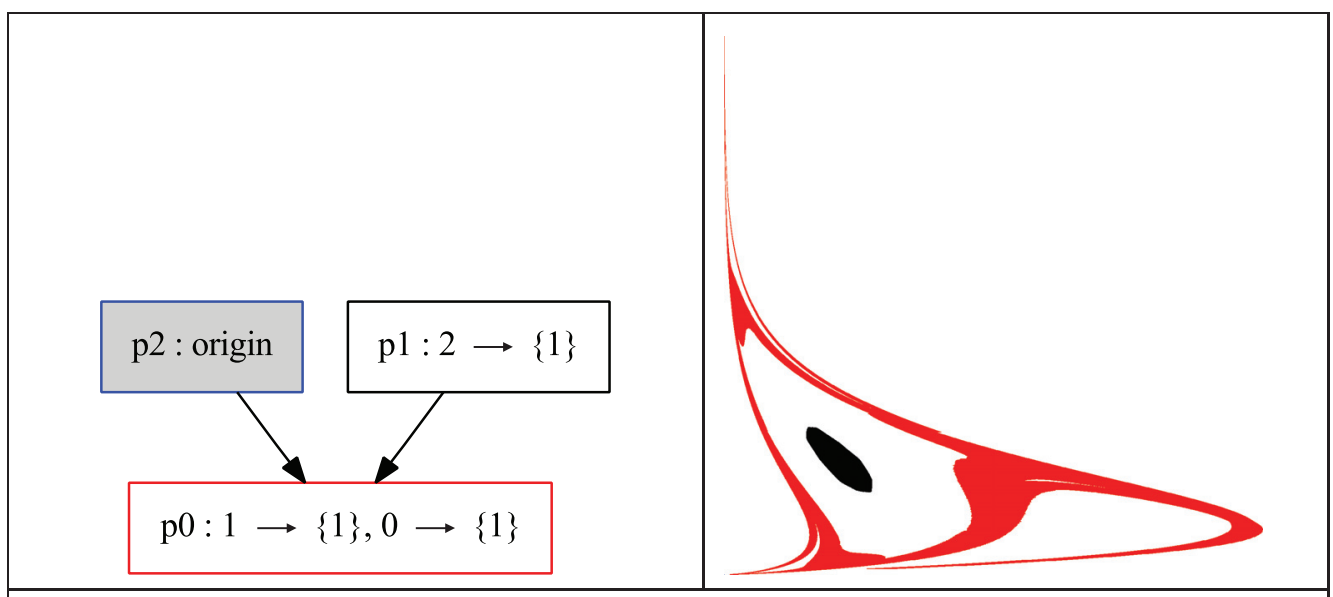

1. The Conley-Morse graph $\mathrm{CMG}(1)$ and the sets $\mathcal{M}(p)$ at the box $(35,32)$.

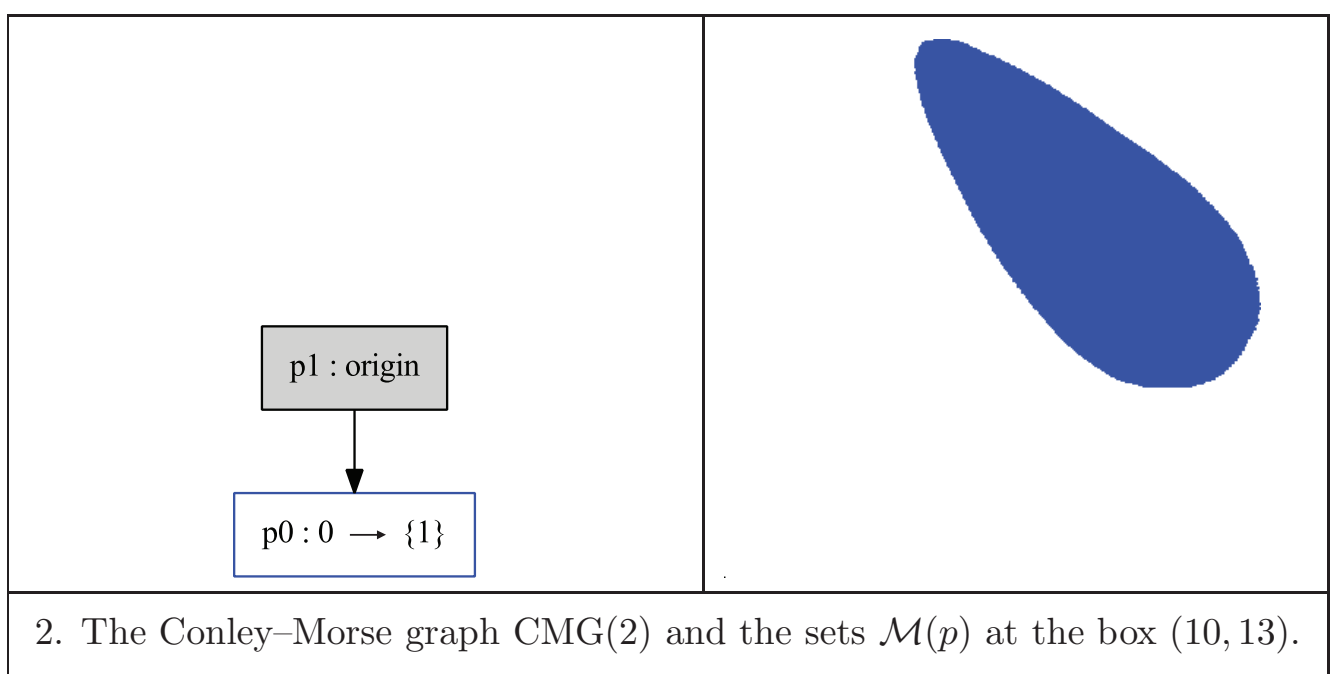

Copyright (c) by SIAM. Unauthorized reproduction of this article is prohibited. 


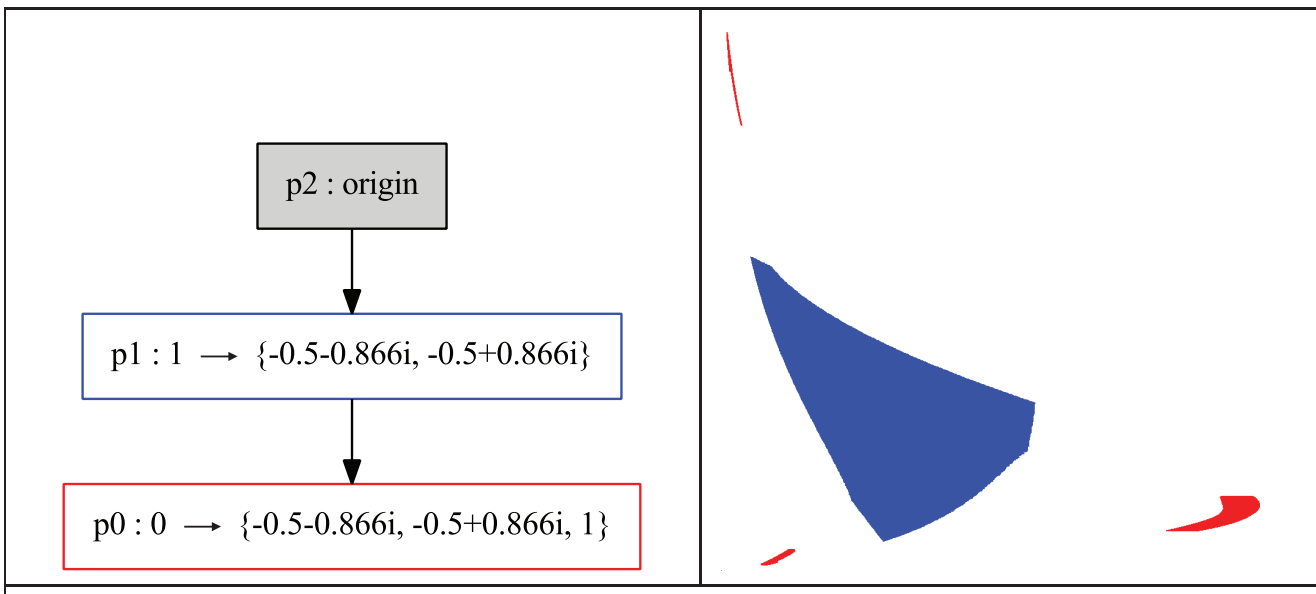

3. The Conley-Morse graph $\operatorname{CMG}(3)$ and the sets $\mathcal{M}(p)$ at the box $(18,12)$.

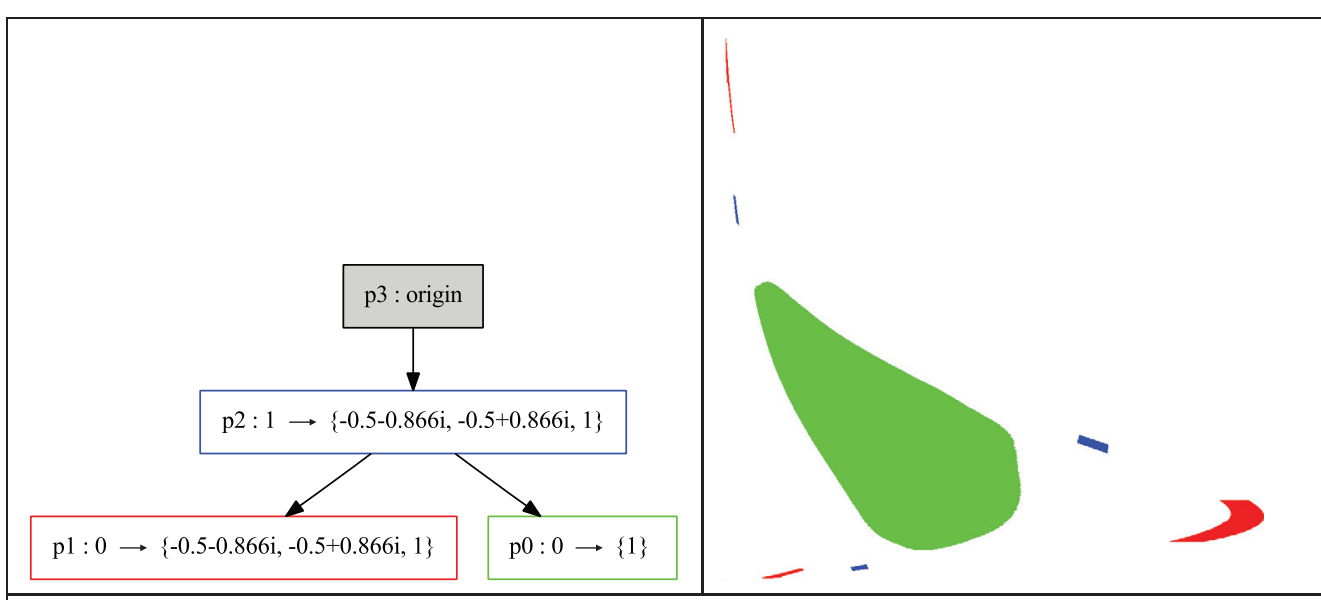

4. The Conley-Morse graph $\mathrm{CMG}(4)$ and the sets $\mathcal{M}(p)$ at the box $(20,22)$.

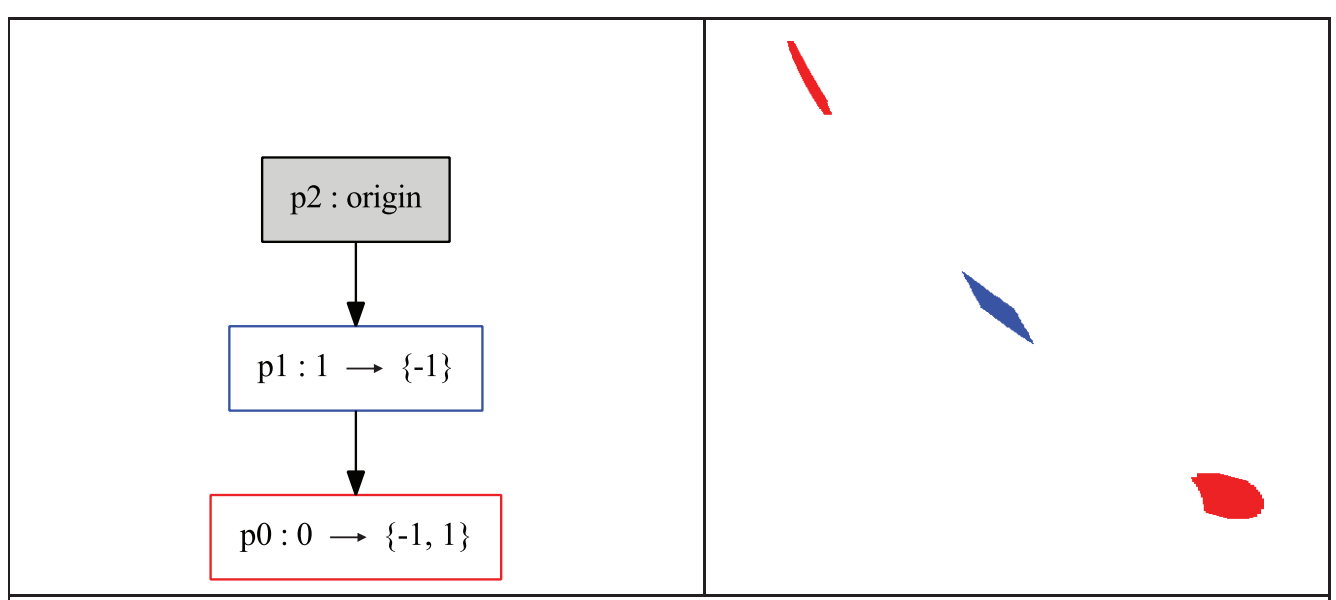

5. The Conley-Morse graph $\mathrm{CMG}(5)$ and the sets $\mathcal{M}(p)$ at the box $(0,45)$. 


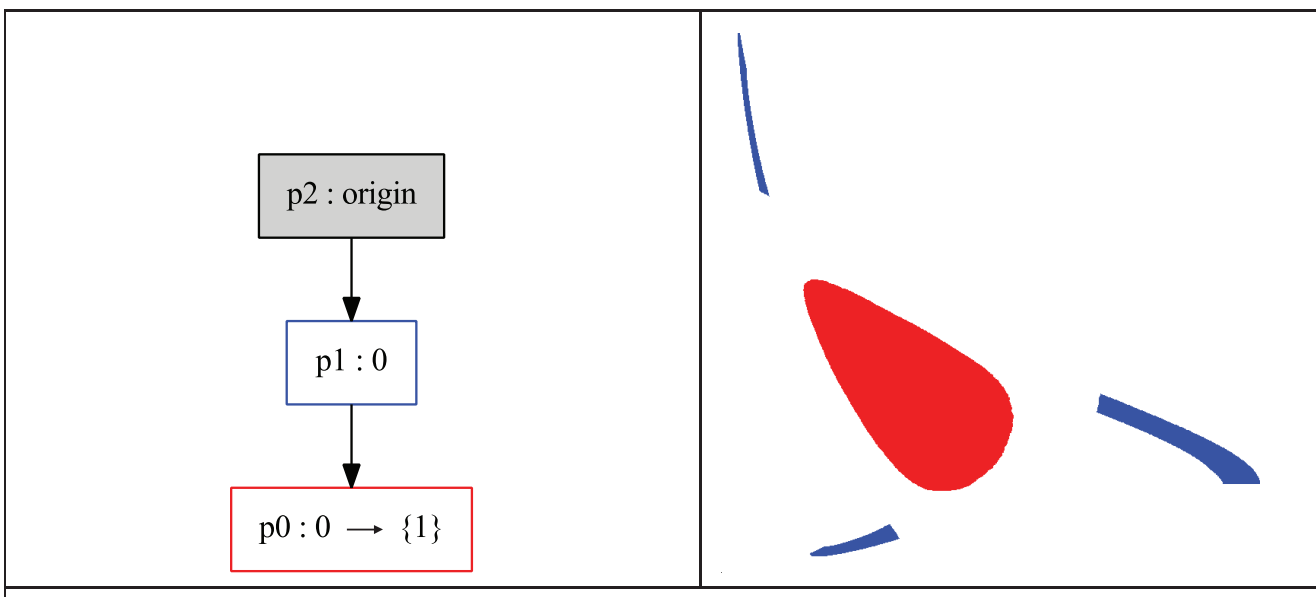

6. The Conley-Morse graph $\mathrm{CMG}(6)$ and the sets $\mathcal{M}(p)$ at the box $(11,11)$.

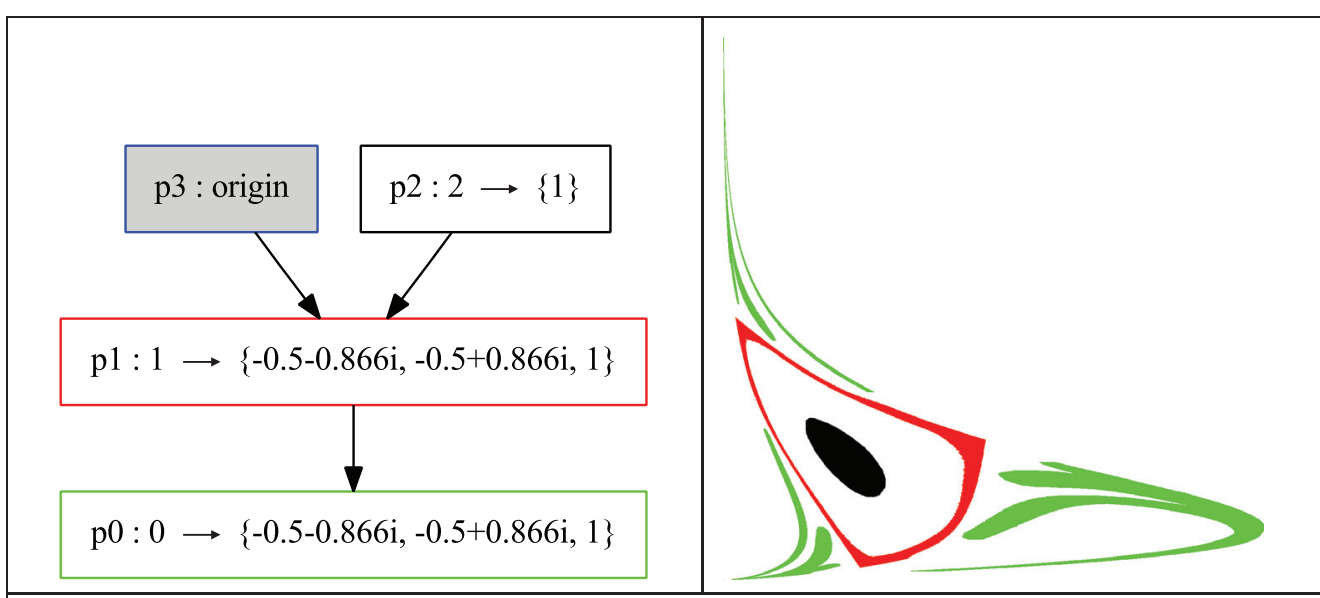

7. The Conley-Morse graph $\mathrm{CMG}(7)$ and the sets $\mathcal{M}(p)$ at the box $(31,22)$.

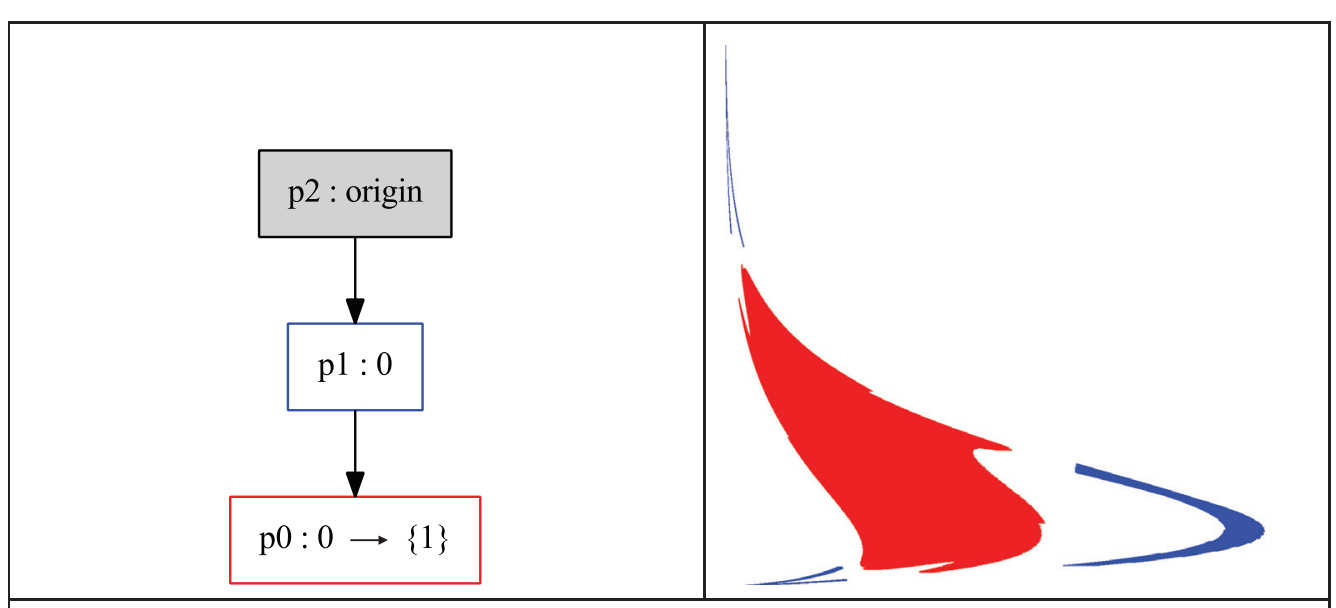

8. The Conley-Morse graph $\mathrm{CMG}(8)$ and the sets $\mathcal{M}(p)$ at the box $(27,39)$. 


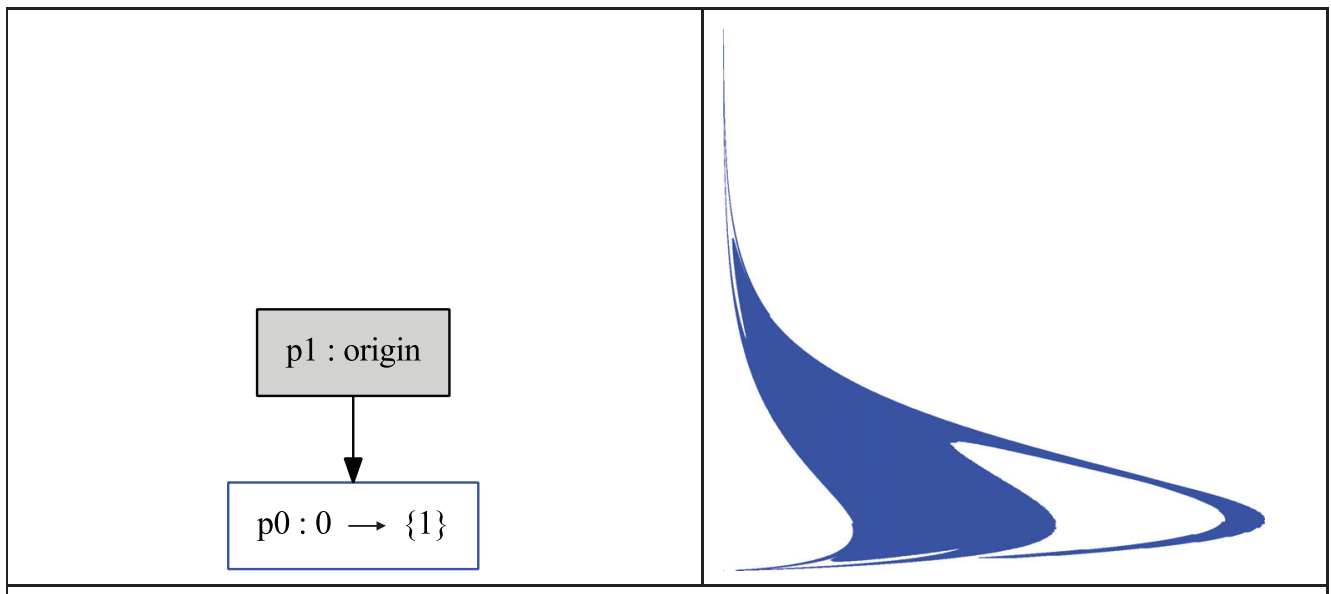

9. The Conley-Morse graph $\mathrm{CMG}(9)$ and the sets $\mathcal{M}(p)$ at the box $(31,47)$.
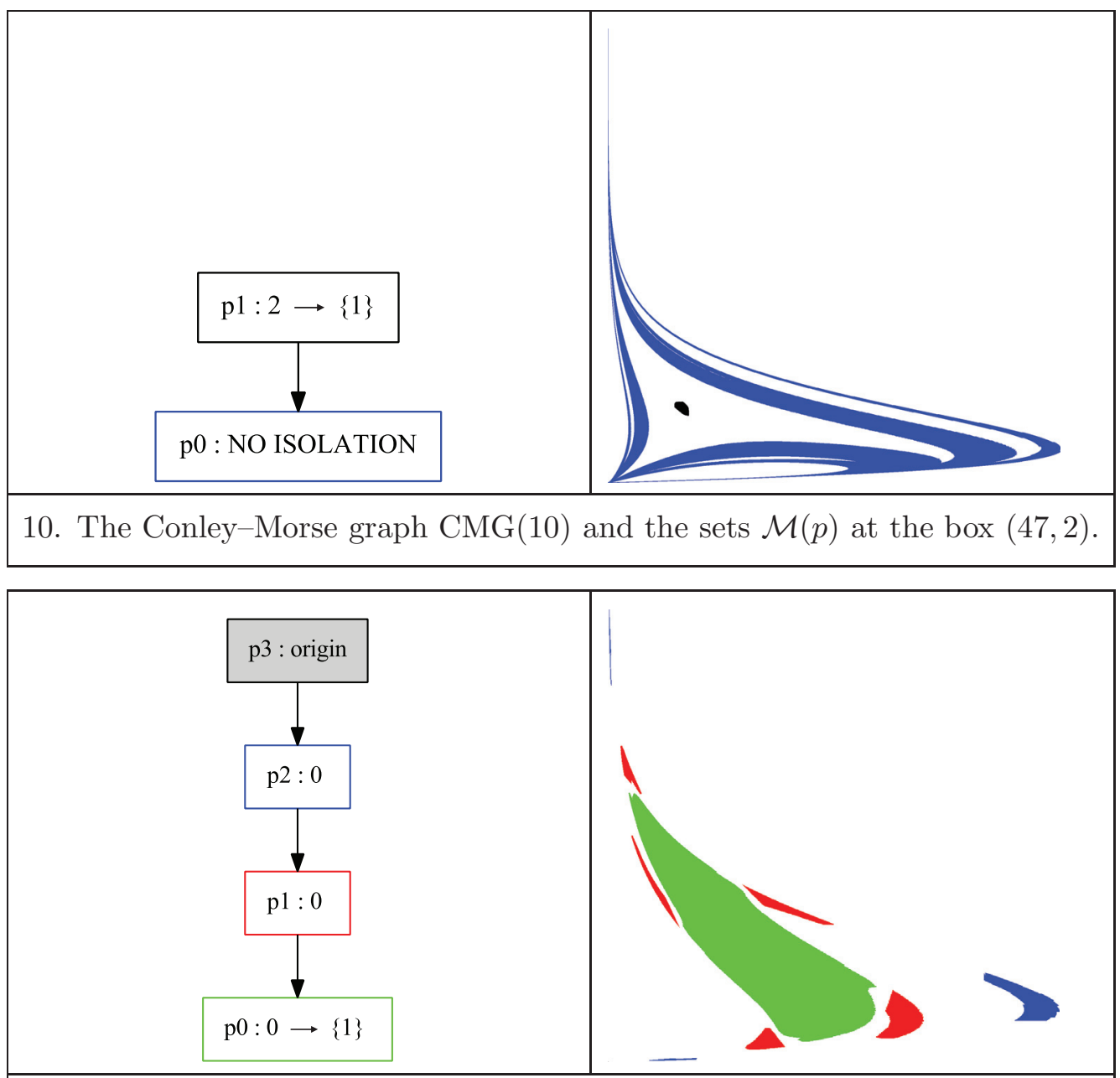

11. The Conley-Morse graph $\mathrm{CMG}(11)$ and the sets $\mathcal{M}(p)$ at the box $(22,47)$. 


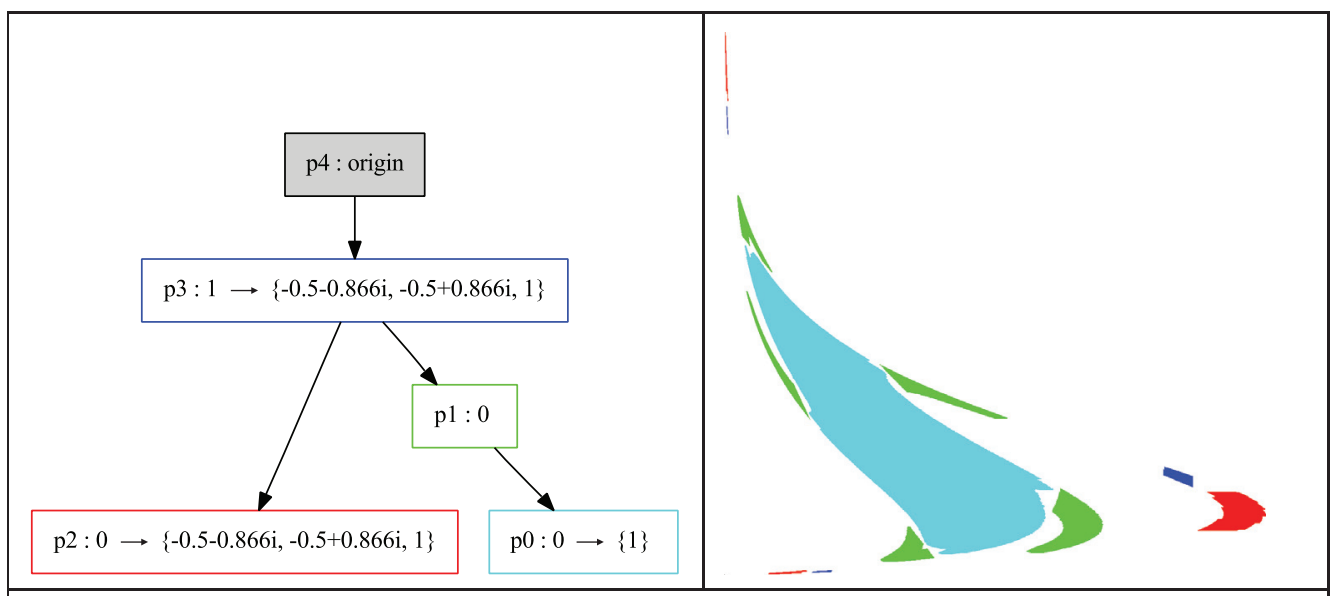

12. The Conley-Morse graph $\mathrm{CMG}(12)$ and the sets $\mathcal{M}(p)$ at the box $(23,49)$.

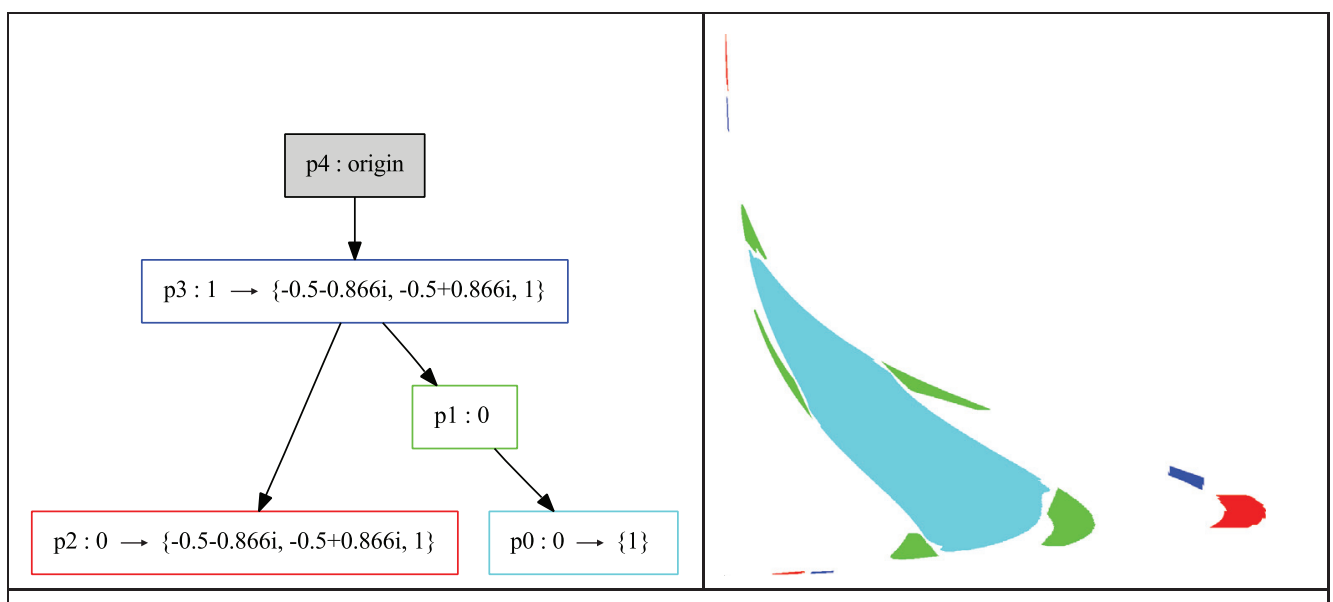

13. The Conley-Morse graph $\mathrm{CMG}(13)$ and the sets $\mathcal{M}(p)$ at the box $(22,45)$.

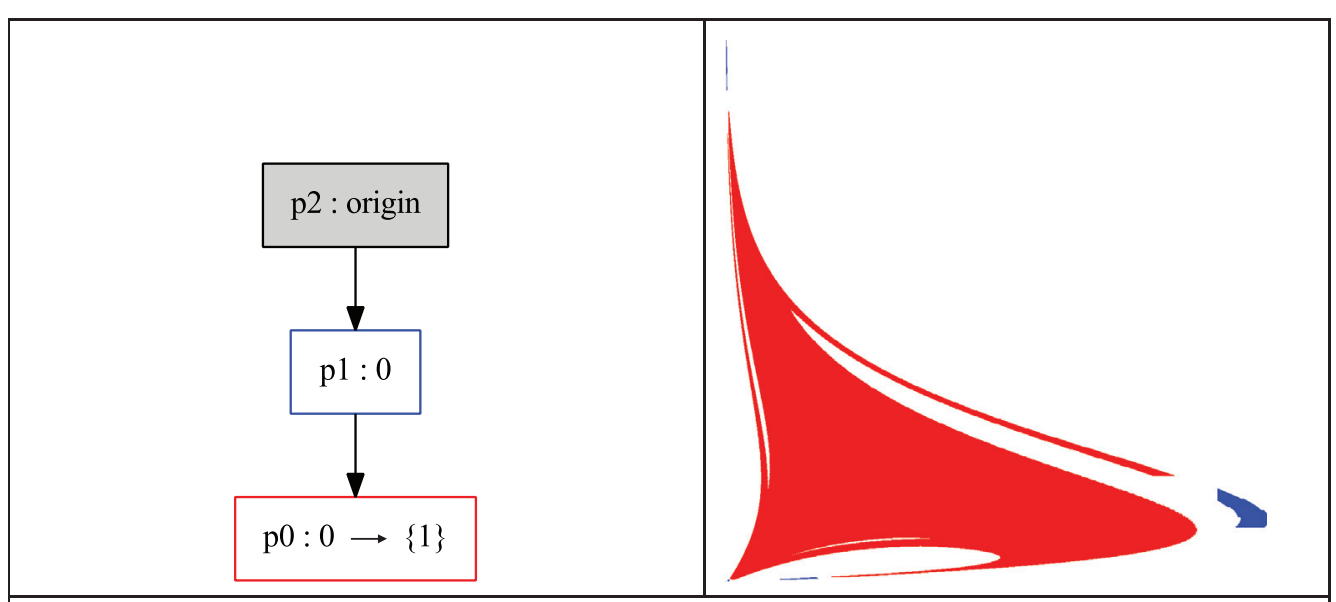

14. The Conley-Morse graph $\mathrm{CMG}(14)$ and the sets $\mathcal{M}(p)$ at the box $(26,0)$. 


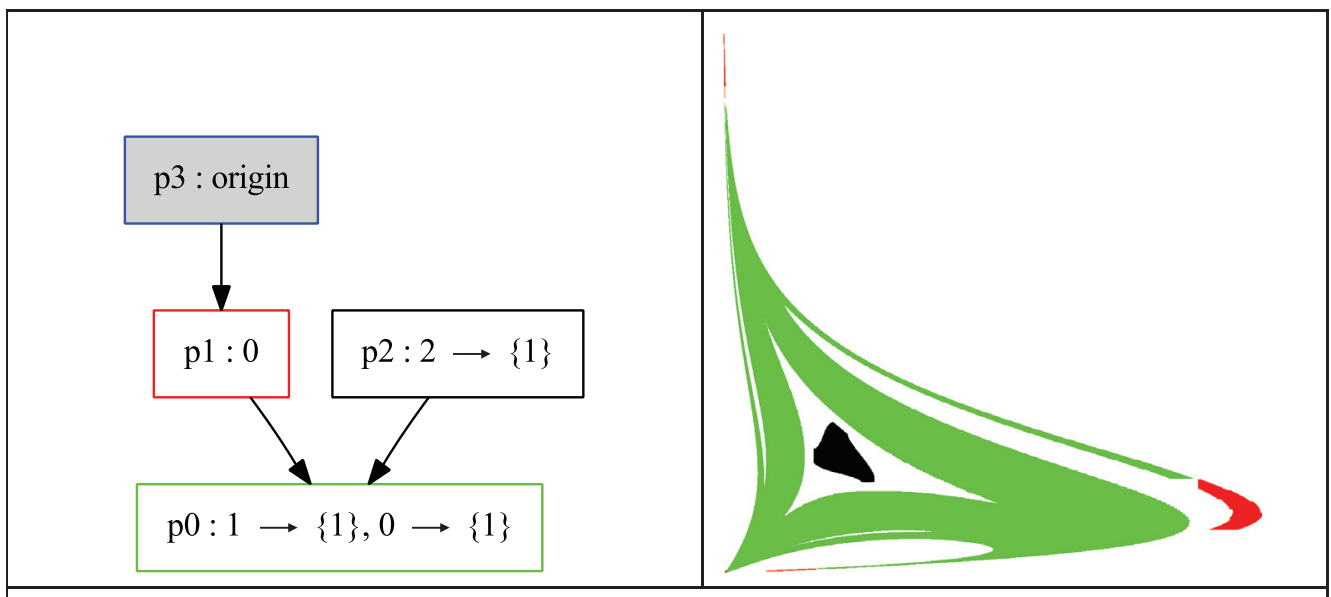

15. The Conley-Morse graph $\mathrm{CMG}(15)$ and the sets $\mathcal{M}(p)$ at the box $(27,0)$.

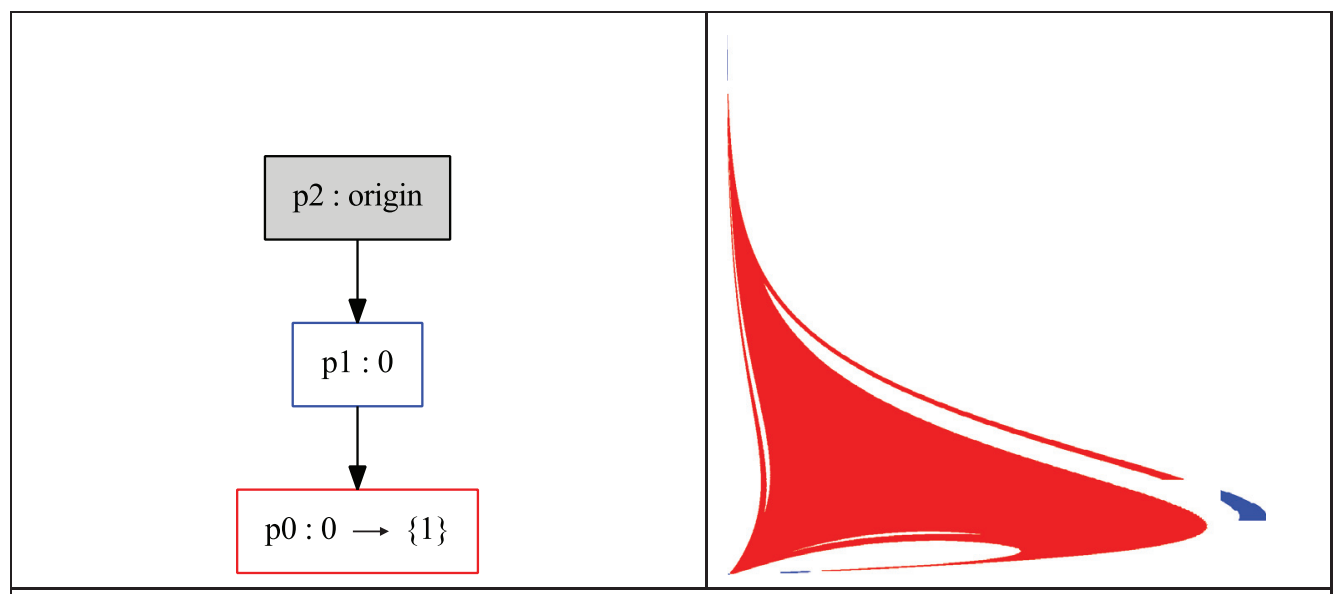

16. The Conley-Morse graph $\mathrm{CMG}(16)$ and the sets $\mathcal{M}(p)$ at the box $(29,1)$.

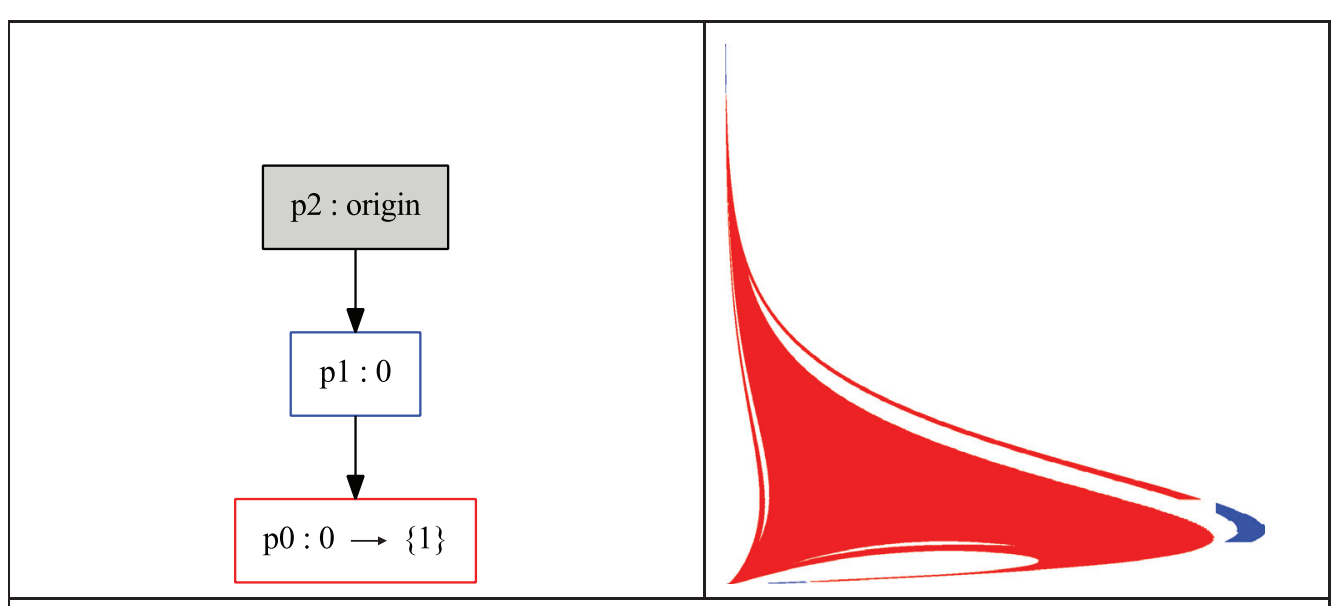

17. The Conley-Morse graph $\mathrm{CMG}(17)$ and the sets $\mathcal{M}(p)$ at the box $(31,2)$. 
5.2. Querying the database. The purpose of the database is to shed light on the possible dynamics exhibited over a wide range of parameter values. To be effective it must be able to be queried. Recall that the database consists of the continuation graph as indicated in Figure 5. Thus, we have the following information at our disposal.

(1) Associated with each node in the continuation graph we have

- the Conley-Morse graph with the information provided in the left panel of the catalogue of continuation classes and

- the set of parameter values $\mathcal{Q}(k)$ associated with the continuation class. (We have not listed $\mathcal{Q}(k)$ explicitly in this paper; however, this information is presented graphically in Figure 6.)

(2) The edges of the continuation graph indicating which continuation classes intersect in parameter space.

Observe that the information in (2) can be recovered from the information in (1). However, we believe it is more efficient to tabulate this information once and store it, as opposed to recomputing it every time that a query is performed.

The reader should also observe that this database is reasonably small. The continuation graph has 17 nodes and 33 edges. Each node contains a directed graph, but these directed graphs typically have only 3 or 4 nodes and edges. Thus, memory is not an issue with regard to storage of the database, and queries of the database are fast. For the remainder of this section we demonstrate how the database can be used to answer relevant questions about the dynamics of the Leslie model.

5.2.1. Multiple basins of attraction. A fundamental question for any dynamical system is whether there exist multiple basins of attraction. Our ability to detect basins of attraction is based on the following proposition, which follows from the fact that $\mathcal{F}_{Q}$ is an outer approximation [9].

Proposition 5.3. Assume A1. Furthermore, assume that $S$ is a global attractor for $F$. Let $\left\{\mathcal{M}_{Q}(p) \mid p \in \mathcal{P}_{Q}\right\}$ be the set of combinatorial Morse sets for $\mathcal{F}_{Q}$. If $q$ is minimal with respect to the order $>_{Q}$, that is, $q \ngtr p$ for all $p \in \mathcal{P}_{Q}$, then $\mathcal{M}(q)$ is a trapping region for $F_{Q}$.

With regard to the density-dependent Leslie model, Remark 5.1 implies that $S$ is the global attractor for the dynamics restricted to $\left(\mathbb{R}^{+}\right)^{2}$. Therefore, the existence of multiple disjoint trapping regions in $\left(\mathbb{R}^{+}\right)^{2}$ implies the existence of multiple distinct basins of attraction. Thus the following query identifies regions in parameter space which support multiple basins of attraction.

Which continuation classes have a Conley-Morse graph with more than one minimal element?

The result of this query is $\{\mathcal{Q}(k): k=4,12,13\}$, and each of the graphs has two minimal elements. Thus $\widehat{\mathcal{Q}}:=\bigcup_{k=4,12,13} \mathcal{Q}(k)$ is a region in parameter space for which there exist at least two basins of attraction. From the edges of the connection graph we see that this defines a connected region (we can compute the homology of $\widehat{\mathcal{Q}}$ to conclude that it is contractible). Furthermore, there are 199 boxes in $\widehat{\mathcal{Q}}$, which represents approximately $8 \%$ of parameter space.

Remark 5.4. If we define $\widehat{\Lambda} \subset \Lambda$ to be the set of parameter values at which $f_{\lambda}$ possesses multiple basins of attraction, then $|\widehat{\mathcal{Q}}| \subset \operatorname{int}(\widehat{\Lambda})$. The inclusion follows from the fact that $\widehat{\mathcal{Q}}$ is

Copyright (c) by SIAM. Unauthorized reproduction of this article is prohibited. 
determined by the outer approximation $\mathcal{F}$, and thus if the attractors are too close together, then they cannot be separated by $\mathcal{F}$. However, given that in applications (1.3) is meant to represent a biological population, one would expect noise in the system. Depending on the variance in the noise and the error bounds associated with $\mathcal{F}$, it is possible that $|\widehat{\mathcal{Q}}|$ is a more appropriate measure of the experimentally observable attractors than $\widehat{\Lambda}$.

5.2.2. Persistence. The notion of persistence was introduced to account for the fact that though a population model, such as the density-dependent Leslie model, is deterministic and predicts that it is impossible to have extinction, in practice populations are subject to stochastic perturbations. For a biologically motivated review, see [22], wherein the following three notions of persistence are discussed. We recast these definitions to be specifically directed toward the Leslie model considered here. Recall the sets $B_{\lambda}$ for $\lambda=\left(\theta_{1}, \theta_{2}\right)$ defined in Remark 5.1, and further define

$$
\stackrel{\circ}{B_{\lambda}}:=\left\{\left(x_{1}, x_{2}\right) \in\left(\mathbb{R}^{+}\right)^{2} \mid 0<x_{1} \leq 10\left(\theta_{1}+\theta_{2}\right) e^{-1}, 0<x_{2} \leq 10 p\left(\theta_{1}+\theta_{2}\right) e^{-1}\right\} .
$$

1. $f$ is persistent despite frequent small perturbations if there exist a state $x \in \dot{B}_{\lambda}$ and $\varepsilon>0$ such that there are no $\varepsilon$-chains from $x$ to 0 .

2. $f$ is persistent despite rare large perturbations if the origin is not in the closure of $\bigcup\left\{\omega(x) \mid x \in B_{\lambda}\right\}$.

3. $f$ is robustly persistent despite frequent small perturbations (respectively, robustly persistent despite rare large perturbations) if all maps $g$ sufficiently near $f$ are persistent despite frequent small perturbations (respectively, persistent despite rare large perturbations).

It is easy to check that $f^{2}\left(B_{\lambda}, \lambda\right) \subset B_{\lambda} \cup\{0\}$. Furthermore, by Remark 5.2 for the parameter range $\Lambda$ covered by the database, the origin is always unstable, and thus for each $\lambda \in \Lambda$ the Leslie model satisfies all these notions of persistence (if one considers $C^{1}$ perturbations of the model).

This statement is no longer true, however, if one fixes the size of the allowed perturbations. This can be seen from the database via the following query:

Which continuation classes have a Conley-Morse graph $\mathrm{CMG}(k)$ in which the box $G$ containing the origin belongs to a combinatorial Morse set $\mathcal{M}(p)$ which is a minimal element of $\mathrm{CMG}(k)$ ?

Observe that for $\mathrm{CMG}(10)$ the minimal Morse set is shaded indicating that the box containing the origin belongs to $\mathcal{M}(0)$. Observe that $\mathcal{Q}(10)$ contains 43 boxes of parameter space and is in the region of the parameter space $\Lambda$ corresponding to large values of seed production of the first age class.

5.2.3. Cycle sets. One of the goals of population dynamics is to explain fluctuations in population levels. We now explain how the database can be used for this purpose. As indicated in section 2.2, the Conley index can be used to understand the structure of the dynamics within a Morse set. For a more complete description of how this can be done, the reader is referred to $[8,13]$. Here we concentrate on the existence of equilibria and periodic orbits. We begin by stating the following result (see $[8,13])$.

Copyright ( $\odot$ by SIAM. Unauthorized reproduction of this article is prohibited. 
Proposition 5.5. Let $f: \mathbb{R}^{n} \rightarrow \mathbb{R}^{n}$ be continuous. If $K$ is a hyperbolic periodic orbit of $f$ with minimal period $\tau \in \mathbb{N}$ and unstable manifold of dimension $d$, then the set of nonzero eigenvalues of the index map occur on the dth homology groups and are

$$
\text { either }\left\{e^{2 \pi i \frac{n}{\tau}} \mid n=0, \ldots, \tau-1\right\} \quad \text { or } \quad\left\{-e^{2 \pi i \frac{n}{\tau}} \mid n=0, \ldots, \tau-1\right\} .
$$

The first case occurs if the action on the unstable manifold is orientation preserving and the second case occurs if the action is orientation reversing.

Remark 5.6. The converse of Proposition 5.5 need not be true. A simple counterexample can be found by considering the logistic equation $f_{\lambda}(x)=\lambda x(1-x)$ for $\lambda \approx 3.0$ and the isolating neighborhood $N=[0.6,0.7]$. For $\lambda<3.0$, the maximal invariant set is a unique stable hyperbolic fixed point, and thus the nonzero eigenvalues are $\{1\}$ on the 0th homology group. However, for $\lambda>3.0$ the maximal invariant set contains an unstable fixed point, a stable periodic orbit of period two, and a connecting orbit from the fixed point to the periodic orbit. Consider, however, the logistic map as a population model with a small amount of noise. At parameter values $\lambda \approx 3.0$, an observer would detect that orbits converge to the neighborhood $N$ but would not be able to distinguish the existence of the period 2 orbit. Thus, from the point of view of an experimentalist, it is conceivable that it is more useful to identify the invariant set inside $N$ with a fixed point. This leads us to the following definition.

Definition 5.7. Let $g: Z \rightarrow Z$ be a continuous map. An isolated invariant set $S$ is a $T$ cycle set if there exist $T$ disjoint, compact regions $N_{1}, \ldots, N_{T}$ such that $S=\operatorname{Inv}(N, g)$, where $N:=\bigcup_{i=1}^{T} N_{i}$ is an isolating neighborhood, and

$$
g\left(N_{i}\right) \cap N \subset N_{i+1}, \quad i=0, \ldots T-1,
$$

where $N_{0}=N_{T}$. Moreover, $S$ is an attracting $T$-cycle set if $g\left(N_{i}\right) \subset N_{i+1}$ for $i=0, \ldots, T-1$.

Heuristically the dynamics associated with a $T$-cycle set could resemble that of a periodic orbit with minimal period $T$, but subject to perturbations.

Proposition 5.8. Let $\mathcal{M}_{Q}$ be a combinatorial Morse set obtained using a rectangular grid on the phase space. Let $M_{Q}=\operatorname{Inv}\left(\left|\mathcal{M}_{Q}\right|, F_{Q}\right)$. Then the set of nontrivial eigenvalues of the index map on the 0 th level of homology is

$$
\text { either } \emptyset \quad \text { or } \quad\left\{e^{2 \pi i \frac{k}{T}} \mid k=0, \ldots, T-1\right\} \text {. }
$$

In the latter case, $M_{Q}$ is an attracting $T$-cycle set.

Proof. Since $\left|\mathcal{M}_{Q}\right|$ is an isolating block [9], the work of [18] implies that there exists an index pair for $F_{Q}$ of the form $P=\left(P_{1}, P_{0}\right)$, where $P_{1}=\left|\mathcal{M}_{Q}\right|$.

Let $\mathcal{M}_{Q}=\bigcup_{j=1}^{J} \mathcal{N}_{j}$, where $\left|\mathcal{N}_{j}\right|$ are the disjoint components of $P_{1}$. Let $\mathcal{J}=\{1, \ldots, J\}$. Let $\mathcal{I}=\left\{j \in \mathcal{J}|| \mathcal{N}_{j} \mid \cap P_{0}=\emptyset\right\}$. Observe that

$$
H_{0}\left(N_{1}, N_{0}\right) \cong \bigoplus_{j \in \mathcal{J}} H_{0}\left(\left|\mathcal{N}_{j}\right|,\left|\mathcal{N}_{j}\right| \cap P_{0}\right) \cong \bigoplus_{j \in \mathcal{I}} H_{0}\left(\left|\mathcal{N}_{j}\right|,\left|\mathcal{N}_{j}\right| \cap P_{0}\right) \cong \bigoplus_{j \in \mathcal{I}} \mathbb{Z}\left[\xi_{j}\right]
$$

where $\xi_{j}$ is the generator of $H_{0}\left(\left|\mathcal{N}_{j}\right|,\left|\mathcal{N}_{j}\right| \cap P_{0}\right)$. 
Consider $j \in \mathcal{I}$. Since $\left(P_{1}, P_{0}\right)$ is an index pair and $\left|\mathcal{N}_{j}\right|$ has no exit set associated with it, this implies that $F_{Q}\left(\left|\mathcal{N}_{j}\right|\right) \subset\left|\mathcal{N}_{\ell}\right|$ for some $\ell \in \mathcal{J}$. If $\ell \in \mathcal{I}$, then $F_{Q P *}\left(\xi_{j}\right)=\xi_{\ell}$. If $\ell \notin \mathcal{I}$, then $F_{Q P *}\left(\xi_{j}\right)=0$.

Since $\mathcal{M}_{Q}$ is a combinatorial Morse set, it is an equivalence class of the recurrent set. Thus, if $\mathcal{I} \neq \mathcal{J}$, then $F_{Q *}$ is nilpotent on the 0 th level. In this case the set of nonzero eigenvalues is $\emptyset$. If $\mathcal{I}=\mathcal{J}$, then $F_{Q P *}$ restricted to the 0 th level is a permutation matrix and the associated eigenvalues are roots of unit with $T=J$.

This remark leads us to propose the following query for identifying attracting cyclic sets.

For a fixed $\tau \in\{1,2,3, \ldots\}$, which continuation classes have a Conley-Morse graph whose minimal node has nonzero eigenvalues $\left\{e^{2 \pi i \frac{n}{\tau}} \mid n=0, \ldots, \tau-1\right\}$ at the 0th homology level?

The result of this query indicates that there is an attracting 1-cycle set for the parameter values $\mathcal{Q}(k), k=1,2,4,6,8,9,11,12,13,14,16,17$, an attracting 2 -cycle set at $\mathcal{Q}(k), k=5$, and an attracting 3 -cycle set at $\mathcal{Q}(k), k=3,4,7,12,13,15$.

Observe that this analysis also provides us with a better understanding of the possible dynamics at those parameter values where we identified multiple basins of attraction $\mathcal{Q}(k)$, $k=4,12,13$. A $T$-cycle set is a very weak description of the dynamics. Careful numerical studies such as those of [25] suggest the existence of chaotic dynamics at some of the parameter values associated with the 3 -cycle sets. Some of this finer information can be also obtained using the Conley index. For example, each 2-cycle and 3-cycle set contains a period 2 and period 3 orbit, respectively (though these orbits need not be stable). There are also Conley index techniques for extracting entropy estimates and symbolic dynamics within Morse sets $[11,23,5,4]$. The relevance of this information is problem-dependent. However, to extract this information will require refinements of the current algorithms being used to construct the database.

As discussed in [3], attempting to match the dynamics of a deterministic model to experimental data in which the presence of noise is to be expected requires an understanding of not only the structure of attractors but also the unstable invariant sets. This is because with sufficient time stochastic events will almost certainly push the trajectory away from the attractors, in which case the dynamics will be determined by the stable and unstable manifolds of the unstable invariant sets. In the context of the Leslie model, perhaps the most interesting cases in which to study the unstable invariant sets is to identify the separatrices in the case of multiple basins of attraction. This is done using the following type of query.

$$
\begin{aligned}
& \text { The minimal nodes in } \mathrm{CMG}(4) \text { are } 1,0 \text {. Find the minimal node } p \text { such that } \\
& p>1 \text { and } p>0 \text {. }
\end{aligned}
$$

The result of this query is $M_{\mathcal{Q}(4)}(2)$ for which the nonzero eigenvalues are $\left\{e^{2 \pi i \frac{n}{3}} \mid n=0,1,2\right\}$, which suggests the behavior of a period 3 orbit with a one-dimensional orientable unstable manifold.

6. Database for the Leslie model. To some extent section 5 is included for pedagogical purposes. Our hope is that the continuation diagram, Figure 6, and the fact that there are only 17 continuation classes provide the reader with a clear indication of the potential use and applicability of the database. As discussed in the derivation of (1.3), the density-dependent 
Leslie model involves three parameters. The computational procedures described in section 4 are dimension-independent, and thus we can apply them to (1.3) over the parameter space

$$
\Lambda:=\left\{\left(\theta_{1}, \theta_{2}, p\right) \in[8,37] \times[3,50] \times[0.5,0.9]\right\} .
$$

We choose an equipartitioned $80 \times 80 \times 40$ grid for this parameter space, given by (4.1) with $m=3, b_{1}=8, b_{2}=3, b_{3}=0.5, \zeta_{1}=29, \zeta_{2}=47, \zeta_{3}=0.4, K_{1}=K_{2}=80$, and $K_{3}=40$.

Recasting the Leslie model in the general form of (1.2) and recalling that $x_{1}$ and $x_{2}$ represent population sizes, we are interested in studying $F:\left(\mathbb{R}^{+}\right)^{2} \times \Lambda \rightarrow\left(\mathbb{R}^{+}\right)^{2} \times \Lambda$, where $\mathbb{R}^{+}:=[0, \infty)$. Remarks 5.1 and 5.2 are still applicable, and thus, as explained in the previous section, for all parameter values $\lambda \in \Lambda$ we compute on

$$
R:=[-0.001,320.056] \times[-0.001,288.051],
$$

and the grids $\mathcal{X}^{(d)}$ in $X=\mathbb{R}^{2}$ are given for $d=1,2, \ldots$ by (4.2) with $n=2, a_{1}=a_{2}=-0.001$, $\xi_{1}=320.057$, and $\xi_{2}=288.052$.

With this input we compute the continuation graph using iteratively generated grids $\mathcal{X}^{(d)}$ in the phase space for $d=6, \ldots, 12$.

Using the techniques outlined in section 4, a continuation graph was computed in 5,225 CPU hours on a cluster based on AMD Opteron 248 processors with 4 GB of memory per node. This results in a continuation graph with 92 vertices and 263 edges. Because of the size of the graph, its visual presentation is of limited use. Also, since we are working with a three-dimensional parameter space and 256,000 parameter boxes, there is no hope of presenting a continuation diagram in this paper. An attempt has been made at the Web site http://chomp.rutgers.edu/database/ to use a series of two-dimensional pictures of slices of the diagram, either as an animation or as a list of consecutive images, to visualize the continuation diagram; an interested reader is kindly invited to explore these results further. Although careful analysis of this kind of visualization may shed light on the dynamics of interest, we would like to point out that this approach will not work in higher dimensions and is therefore of limited use.

Independent of how complicated the continuation graph is, however, the queries from section 5.2 can still be performed with equal ease and provide the following results:

- There are parameter values associated with multiple basins of attraction.

- At $\mathcal{Q}(k), k=6,10,34,37,51,58,59$ (comprising 18,632 boxes in parameter space), one attractor is a 1-cycle set and the other attractor is a 3 -cycle set. The index information suggests (there is no theorem to guarantee this; however, visual inspection confirms this) that the separatrix between the basins of attraction has the behavior of an unstable 3 -cycle set.

- At $\mathcal{Q}(k), k=15,17$ (comprising 221 boxes in parameter space), one attractor is a 2-cycle set and the other attractor is a 3-cycle set. The index information also suggests that the separatrix between the basins of attraction has the behavior of an unstable 3-cycle set.

- Persistence is lost on the level of the computational grid for the parameter values in $\mathcal{Q}(k), k=7$ (comprising 16,686 boxes in parameter space).

Copyright $\odot$ by SIAM. Unauthorized reproduction of this article is prohibited. 
7. Final remarks. The presentation of this paper has focused on discrete time dynamical systems. On a theoretical level it is clear that the same ideas apply to dynamics generated by differential equations. In particular, if one considers a parameterized family of semiflows $\varphi:[0, \infty) \times X \times \Lambda \rightarrow X$ and chooses a constant $\tau>0$, then the time- $\tau$ map $\varphi_{\tau}=\varphi(\tau, \cdot, \cdot): X \times \Lambda \rightarrow X$ returns one to the setting of this paper. On a practical level the issue is much more subtle. Typically, the semiflow is defined in terms of solutions to a differential equation. Thus, constructing an outer approximation requires rigorous numerical calculation of an enclosure of the forward translation of a grid element. There are tools for doing this effectively (see, in particular, the work of the CAPD group [26]); however, the computational cost is clearly much greater than the simple evaluation of a formula, as was done in this paper. Furthermore, if $\tau$ is chosen to be small, then $\varphi_{\tau}$ represents a perturbation of the identity. In order to identify interesting dynamics in this case, the diameter of the grid on phase space will need to be extremely small. On the other hand, if $\tau$ is chosen to be large, then not only does the cost of evaluation of $\varphi_{\tau}$ on grid elements become large, growing at least proportionally to $\tau$, but also for a reasonable computational cost, the error bounds on the images of the grid elements will be extremely large. An important open problem is then how to choose, a priori, an appropriate size for $\tau$.

Another obvious topic which has been only briefly discussed is the relationship between the continuation classes and classical bifurcation theory. As was pointed out in Remark 3.8, the boundaries between the continuation classes need not represent boundaries between different topological conjugacy classes of dynamics, and vice versa. Using standard bifurcation analysis it is fairly easy to check that a period-doubling bifurcation occurs near the boundary between continuation classes 2 and 5 (see Figure 6). Similarly, a saddle-node bifurcation to a period 3 orbit occurs within continuation class 6 as one passes from continuation class 2 to continuation class 4. However, recognizing these bifurcations from the database and predicting how the database will represent these bifurcations as a function of grid size in both phase space and parameter space remains an open problem.

Acknowledgment. The authors thank anonymous referees for their careful reading of the manuscript and their useful suggestions.

\section{REFERENCES}

[1] H. BAn And W. Kalies, A computational approach to Conley's decomposition theorem, J. Comput. Nonlinear Dynam., 1 (2006), pp. 312-319.

[2] C. Conley, Isolated Invariant Sets and the Morse Index, CBMS Regional Conference Series in Mathematics 38, AMS, Providence, RI, 1978.

[3] J. M. Cushing, R. F. Costantino, B. Dennis, R. A. Desharnais, and S. M. Henson, Nonlinear population dynamics: Models, experiments and data, J. Theoret. Biol., 194 (1998), pp. 1-9.

[4] S. Day, R. Frongillo, And R. Treviño, Algorithms for rigorous entropy bounds and symbolic dynamics, SIAM J. Appl. Dyn. Syst., 7 (2008), pp. 1477-1506.

[5] S. Day, O. Junge, And K. Mischaikow, A rigorous numerical method for the global analysis of infinitedimensional discrete dynamical systems, SIAM J. Appl. Dyn. Syst., 3 (2004), pp. 117-160.

[6] M. Dellnitz, G. Froyland, and O. Junge, The algorithms behind GAIO—set oriented numerical methods for dynamical systems, in Ergodic Theory, Analysis, and Efficient Simulation of Dynamical Systems, B. Fiedler, ed., Springer-Verlag, New York, 2001, pp. 145-174.

[7] M. Dellnitz And A. Hohmann, A subdivision algorithm for the computation of unstable manifolds and global attractors, Numer. Math., 75 (1997), pp. 293-317.

Copyright (c) by SIAM. Unauthorized reproduction of this article is prohibited. 
[8] T. Kaczynski, K. Mischaikow, and M. Mrozek, Computational Homology, Appl. Math. Sci. 157, Springer-Verlag, New York, 2004.

[9] W. D. Kalies, K. Mischaikow, and R. C. A. M. VanderVorst, An algorithmic approach to chain recurrence, Found. Comput. Math., 5 (2005), pp. 409-449.

[10] A. LAnder, A calculus of purpose, PLoS Biology, 2 (2004), pp. 712-714.

[11] K. Mischaikow And M. Mrozek, Isolating neighborhoods and chaos, Japan J. Indust. Appl. Math., 12 (1995), pp. 205-236.

[12] K. Mischaikow And M. MrozeK, Chaos in the Lorenz equations: A computer-assisted proof, Bull. Amer. Math. Soc. (N.S.), 32 (1995), pp. 66-72.

[13] K. Mischaikow and M. Mrozek, Conley index, in Handbook of Dynamical Systems, Vol. 2, NorthHolland, Amsterdam, 2002, pp. 393-460.

[14] K. Mischaikow, M. Mrozek, and P. Pilarczyk, Graph approach to the computation of the homology of continuous maps, Found. Comput. Math., 5 (2005), pp. 199-229.

[15] J. Montgomery, Cohomology of isolated invariant sets under perturbation, J. Differential Equations, 13 (1973), pp. 257-299.

[16] R. E. Moore, Interval Analysis, Prentice-Hall, Englewood Cliffs, NJ, 1966.

[17] M. MrozeK, An algorithm approach to the Conley index theory, J. Dynam. Differential Equations, 11 (1999), pp. 711-734.

[18] M. MrozeK, Index pairs algorithms, Found. Comput. Math., 6 (2006), pp. 457-493.

[19] P. PilARCZYK, A concurrent algorithm for the construction of Conley index pairs, in preparation.

[20] P. PilarczyK And K. Stolot, Excision-preserving cubical approach to the algorithmic computation of the discrete Conley index, Topology Appl., 155 (2008), pp. 1149-1162.

[21] J. W. Robbin And D. Salamon, Dynamical systems, shape theory and the Conley index, Ergodic Theory Dynam. Systems, 8 (1988), pp. 375-393.

[22] S. J. SchreiBer, Persistence despite perturbations for interacting populations, J. Theoret. Biol., 242 (2006), pp. 844-852.

[23] A. Szymczak, The Conley index and symbolic dynamics, Topology, 35 (1996), pp. 287-299.

[24] A. Szymczak, A combinatorial procedure for finding isolating neighborhoods and index pairs, Proc. Roy. Soc. Edinburgh Sect. A, 127 (1997), pp. 1075-1088.

[25] I. Ugarcovici And H. Weiss, Chaotic dynamics of a nonlinear density dependent population model, Nonlinearity, 17 (2004), pp. 1689-1711.

[26] Computer Assisted Proofs in Dynamics group, http://capd.wsb-nlu.edu.pl/.

Copyright (C) by SIAM. Unauthorized reproduction of this article is prohibited. 